\title{
Edition of IGI 2
}

1 šumma amēlu īnāšu murdinnī malâ sahlê qēm labti ina šikari tarabbak tașammid UD.3.KAM ìnišu [lā tapattar]

2 [kala] ūme šalāšššs tarakkas ina rebî ūmi ina suādi erri īnī̌u takaddad mê ina? [libbi? īnī̌̌u tanaddi]

3 [īnišu] tušakkal qerša emma ištiššu šinišu šalāšišu īnišu tarakkas lipi kursinni șabīti inišš [...] ...

4 [ina] arkīti ina irri abāri tasâk īnī̌u teqqi hūrata tasâk ana muhhi īnī̌u tābīla tazarru tașammid

5 kala ūme šalāšǐ̌̌ riksa ukâlma inassah kalbāna ${ }^{\text {(gloss: ü bina) }} z \bar{e} r q u d r i$ burāša

6 labta sahlê taballal tābìla ana muhhi qaqqadišu tazarru qaqqassu tașammidma UD.3.KAM [lā] tapațtar

7 ina rebî ūme tapațțaršuma qaqqassu tugallab šamma ribka īnīš tēteneqqi ina'eš
'If a man's eyes are full of murdinnubrambles: you shall make an infusion from sahlû-plant (and) flour of roasted grain in beer, (and) bandage (his eyes). For three days [you do not loosen (the bandage from)] his eyes. ${ }^{2}$ [Every] day you bandage (his eyes) three times. On the fourth day, you rub his eyes with parched suādu-aromatic, [(and) you put] water into? [his eyes.] ${ }^{3}$ You wipe [his eyes], (and) bandage his eyes once, twice, (and) three times with a hot strip of meat, (and) you ... [his] eyes with fat from a gazelle's knuckle. ${ }^{4}$ Afterwards you pound (the previous substances) in a lead salve and daub his eyes. You pound a dyeing sumac, spatter it in a dry state over his eyes, (and) bandage (the eyes). ${ }^{5}$ Three times during the whole day he keeps the binding on (his eyes) and then he removes (it). ${ }^{6}$ You mix ${ }^{5}$ kalbānu-plant ${ }^{\text {(gloss: or tamarisk), } \operatorname{seed}(\mathrm{s}) \text { of }}$ qudru-plant, juniper, ${ }^{6}$ roasted grain (and) sahlû-plant. In dry state, you spatter (the ingredients) over his head, you bandage his head and then for three days you do [not] loosen (the bandage). ${ }^{7}$ On the fourth day you release (the bandage) and you shave his head. You keep daubing his eyes with the drug infusion (and) he will get better.

$1 \quad$ ND i $1 \quad$ [............................ mur-di]n-ni DIRI sah-lé-e Zİ ŠE.SA.A ina KAŠ tarabak LAL UD.3.KÁM IGI.MIN-š[ú NU DU $]$

Overview of IGI 2 Manuscripts and Parallels: AA=BAM 20; AB=BAM 159; AD=BAM 22; AF=BAM 23; AG=BAM 15; bB=BAM 382; BoA=KUB 4/50; KA=CTN 4/123; NA=BAM 510; NB=BAM 513; NC=BAM 514; ND=BAM 515; NE=BAM 516; NI=BAM 480; NK=BAM 518; sA=IRAQ 65; $\mathbf{u A}=$ SpTU 50; $\mathbf{u B}=\mathrm{NBC} 4211 ; \mathbf{x A}=\mathrm{CM} 37$

2 Open Access. (c) 2020 Markham J. Geller and Strahil V. Panayotov, published by De Gruyter. (c) BY-NC-ND This work is licensed under the Creative Commons Attribution-NonCommercial-NoDerivatives 4.0 License. 
(NA) iv 47 DIŠ NA IGI.MIN-šú mur-din-n[i DIRI] sah-lé-e Zİ .] KA[ک̌ .......<smiles>[AlH2]</smiles>

2 NDi 2 [ka-la $]^{\ulcorner}$UD-me 3-šú ${ }^{2}$ KEŠDA ina 4 UD-mi ina šm MAN.DU er-ri IGI.MINšú ta-kad-da-ad A ina Š[À IGI.MIN-šú ŠUB]

3 ND i 3 [IGI.MIN-šú t]u-šak-kal qer-šá em-ma 1-šú 2-šú 3-šú IGI.MIN-šú KEŠDA İ.UDU kur-sin-ni MAŠ.DÀ IG[I.MIN-šú (x) ] x-pap?

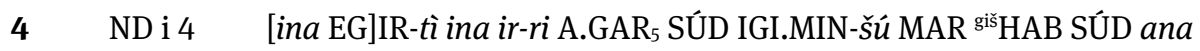
UGU IGI.MIN-šú ta-bi-la $a_{12}{ }^{\ulcorner}$MAR $\urcorner$LAL

5 ND i $5 \quad[k] a-l a$ UD-me 3-šú KEŠDA ú-kal-ma ZI giš̀MAŠ.HUŠ (gloss: u giš.šinig) NUMUN ${ }^{\text {uKI. }}{ }^{\mathrm{d} I S ̌ K U R}{ }^{[\mathrm{[si}] \mathrm{m} ? \mathrm{LI}}$

6 ND i 6 ŠE.SA.A sah-lé-e HE.HE ta-bi-la $a_{12}$ ana UGU SAG.DU-šú MAR SAG.DUsu LAL-ma U[D.3.KÁM NU D]U

7 ND i 7 ina 4 UD-me DU 8 -šú-ma SAG.DU-su tu-gal-lab Ú rib-ka IGI.MIN-šú MAR.MEŠ [ina-e]š

8 šammu peșû rikibti arkabi emesalli aban gabî uhūlu qarnānû murru qadūt [šikāni]

9 kasû naphar samānat šammī țīpu šumma amēlu īnāšu șēta hanțā u murdinnī [malâ]

${ }^{8}$ White plant, bat guano, emesallu-saline solution, alum, horned uhūlu-alkali, bitter-plant, river [silt], ${ }^{9} k a s u \hat{u}$-plant: total of eight drugs, which are (used as) drops, if a man's eyes are inflamed (lit. burn) with sun-heat and [full] of a murdinnu- brambles.

\begin{tabular}{|c|c|c|}
\hline 8 & ND i 8 & 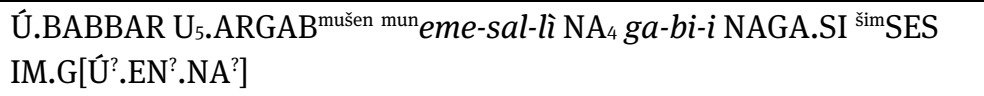 \\
\hline 9 & ND i 9 & $\begin{array}{l}\text { GAZI }^{\text {sar }} \text { PAP } 8 \text { Ú.MEŠ ți } i_{5}-p u \text { šum-ma NA IGI.MIN-šú UD.DA ha-an-ța u } \\
\text { mur-d[in-ni DIRI }]\end{array}$ \\
\hline
\end{tabular}

Overview of IGI 2 Manuscripts and Parallels: AA=BAM 20; AB=BAM 159; AD=BAM 22; AF=BAM 23; AG=BAM 15; bB=BAM 382; BoA=KUB 4/50; KA=CTN 4/123; NA=BAM 510; NB=BAM 513; NC=BAM 514; ND=BAM 515; NE=BAM 516; NI=BAM 480; NK=BAM 518; sA=IRAQ 65; $\mathbf{u A}=\mathrm{SpTU} 50 ; \mathbf{u B}=\mathrm{NBC} 4211 ; \mathbf{x A}=\mathrm{CM} 37$ 
10 šammu peșû aban gabî emesalli annû tīpu [ša ìnī]

Alternative prescription 1

11 kukru qēm labti kasû annû [țīpu ša ìnī]
${ }^{10}$ White plant, alum, emesallu-saline solution; these (drugs) are (used as) drops [for the eyes].

Alternative prescription 1

${ }^{11} \mathrm{Kukru}$-aromatic, flour of roasted grain, kasû-plant; these (drugs) are (used as) [drops for the eyes.]

10 ND i 10 Ú.BABBAR NA 4 ga-bi-i ${ }^{\text {mun }} e m e-s a l-l i ̀ ~ a n-n u-u ́ ~ t i i-[p u$ šá IGI.MIN]

(NK) 8， $\mathrm{NA}_{4}$ g]a-bi-i ${ }^{\text {mun }}$ eme-sal-lì ți $i_{5}-p u$ šá ${ }^{~}$ IGI.MIN.MEŠ`

(AA) 9' Ú.BABBAR NA 4 ga-bi-i ${ }^{\operatorname{mun}}[$ eme-sal-li]

(AA) 10’ an-nu-u tii-pu šá I[GI(.MIN)]

(sA) ii 20 Ú.BABBAR NA 4 ga-bi-i mun? eme-sal-li (AN ATTEMPT TO WRITE THE NUMBER 3?)

(sA) ii 21 Ú.MEŠ ți-pi šá IGI.MIN

$11 \quad$ ND i 11 ršim?GÚR.GÚR Zİ ŠE.SA.A GAZI ${ }^{\mathrm{sar}}$ an-nu-ú [ti-pu šá IGI.MIN]

(AA) 7’ šmGÚR.GÚR Zİ ŠE.SA.A GA[ZI $\left.{ }^{\mathrm{sar}}\right]$

(AA) 8,

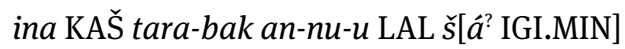

12 [šumma] amēlu ìnāšu giddagiddâ ukallā ana bīti ahî illakma bābu išassi kìma $x[. .$.

13 [...] giddagiddû ašar šanîmma giddagiddûkunuma tablā šanîš [...]

\section{Alternative prescription 1}

14 ana [bìt $\left(\right.$ lă$\left.\left.^{2}\right)\right]$ edê illakma bāba išassi kìma x x x [...]

15 ... giddagiddû ašar šanîmma giddagiddûkunuma tablā ... [...]

${ }^{12}$ If a man's eyes contain giddagiddûfibers, he goes to a stranger's house and calls at the entrance door': 'like x [...] ${ }^{13}[. .$.$] giddagiddû-fibers, ditto, take away$ your giddagiddû-fibers, ditto ...!'

\section{Alternative prescription 1}

${ }^{14} \mathrm{He}$ goes [to] an (un)known [house] and calls at the entrance door: 'like ... [...]. ${ }^{15}$... giddagidd $\hat{u}$-fibers, ditto, remove your (pl.) giddagiddû-fibers, ... [...]!'

12 ND i 12 [DIŠ N]A `IGI.MIN-šú GÍD.DA.GÍD.DA ú?-kal-la ana É $a$-hi-i DU-ma KÁ? GÙ-si ki-ma x [...]

$13 \quad$ ND i 13 $\underline{\text { [ ... GÍD.D]A.GÍD- } d u \text { `KIMIN GÍD.DA.GÍD- } d u\urcorner-k u-n u-m a \text { tab-la MIN [...] }}$

Overview of IGI 2 Manuscripts and Parallels: AA=BAM 20; AB=BAM 159; AD=BAM 22; AF=BAM 23; AG=BAM 15; $\mathbf{b B}=\mathrm{BAM}$ 382; BoA=KUB 4/50; KA=CTN 4/123; NA=BAM 510; NB=BAM 513; NC=BAM 514; ND=BAM 515; NE=BAM 516; NI=BAM 480; NK=BAM 518; sA=IRAQ 65; $\mathbf{u A}=$ SpTU 50; $\mathbf{u B}=\mathrm{NBC} 4211 ; \mathbf{x A}=\mathrm{CM} 37$ 


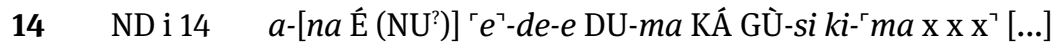

15 ND i 15 HI x [ (x x) GÍ]D.DA.GÍD- $d u$ KIMIN GÍD.GÍD-ku-nu-ma tab-la x [...]

\begin{tabular}{|l|l|}
\hline $\begin{array}{l}\text { šumma amēlu [ina] īnīšu lipištu kukra } \\
\text { burāša țūra zēr bìni [... tasâk .... }\end{array}$ & $\begin{array}{l}{ }^{16} \text { If there is a fleshy substance [in] a man's } \\
\text { eyes; [you pound] kukru-aromatic, } \\
\text { juniper, țūru-plant, seeds of tamarisk... }\end{array}$ \\
\hline
\end{tabular}

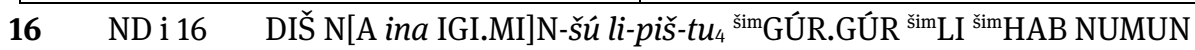
ŠI[NIG ... SÚD ...]

17 šumma amēlu ina īnī̌su šārtu așât u ìnāšu ašâ țābta kamma lipi [kalīti? ...]

18 ša immeri dišip šadê malmališ tasâk ina himēti taballal ìnīš teqqi tamaš[šaš? ...]
${ }^{17}$ If a hair protrudes from a man's eyes, and his eyes are blurred: ${ }^{18}$ you pound in equal amounts ${ }^{17}$ salt, kammu-tanningfungus, [kidney?] fat [...] ${ }^{18}$ of a sheep (and) mountain honey (and) you mix (them) in ghee. You daub his eyes, (and) wipe (them) [...]

17 ND i 17 DIŠ NA in[a IG]I.MIN-šú SÍG $a$-șa-at u IGI.MIN-šú $a$-šáa- $a$ MUN kám-ma 'İ.UDU' [ÉLLAG? ...]

18 ND i 18 šá UDU.NÍ[TA LÀ]L.KUR.RA mál-ma-liš SÚD ina İ.NUN HE.HE IGI.MIN-šú MAR ta-ma[š-aš

19 šumma amēlu ìnāšu šēra ālika malâ ana [bulluțī̌̌ ...]

20 [...zēr] GI.ZÚ.LUM.MA ina dišpi tasâk teqqi : ... [...]

21 [...] ashar ina himēti tasâk teqqi : ... [...]

19 ND i 19 DIŠ N[A IGI.MIN]-šú UZU $a$-li-ka $a_{13}$ DIRI- $a$ ana [TI-šú ...]

20 ND i 20 x [x NUMUN] GI.ZÚ.LUM.MA ina LÀL SÚD MAR : x [...]

21 ND i $21 \quad\left[\mathrm{x} \mathrm{x}^{\mathrm{n}}\right]^{\mathrm{a} 4}$ as-har ina İ.NUN SÚD MAR : x [...]

Overview of IGI 2 Manuscripts and Parallels: AA=BAM 20; AB=BAM 159; AD=BAM 22; AF=BAM 23; AG=BAM 15; bB=BAM 382; BoA=KUB 4/50; KA=CTN 4/123; NA=BAM 510; NB=BAM 513; NC=BAM 514; ND=BAM 515; NE=BAM 516; NI=BAM 480; NK=BAM 518; sA=IRAQ 65; $\mathbf{u A}=\operatorname{SpTU} 50 ; \mathbf{u B}=\mathrm{NBC} 4211 ; \mathbf{x A}=\mathrm{CM} 37$ 
22 [šumma amēlu] ìnāšu šēra ālika malâma u illak ana bulluțǐšu [sahlê nīnâ murra lipâ ...]

23 kanakta šamma peșâ rikibti arkabi [emesalli ištēniš tasâk ...]

24 ina dām erēni lu ina šaman erēni taballal passa teppuš [ina mê tasâk īnāšu teqqi]
${ }^{22}$ [If a man's] eyes are full of loose tissue and it moves, in order to heal it: $\left[{ }^{23}\right.$ you pound together ${ }^{22}$ sahlû-plant, ninû-mint, bitter-plant, fat, ] ${ }^{23}$ kanaktu-aromatic, white plant, bat guano, [emesallu-saline solution, .... . ${ }^{24}$ You mix (them) in cedar 'blood' (i.e. resin) or in cedar oil (and) [you make] a collyrium stick [(out of it), you pound (it) in water and daub his eyes].

22 ND i 22 [DIŠ NA IGI.M]IN-šú UZU DU-ka $a_{16}$ DIRI-ma u DU-ak ana TI- $\ulcorner$ šú $\mathrm{x}$ [...]

(AD) 4f.' DIŠ NA IGI.MIN-šú UZU DU-ka DI[RI-ma DU-k]a ana TI-šú / sah-lé-e úKUR.RA ši[mSE]S İ.UDU $\rightarrow$

23 ND i $23 \quad{ }^{[s ̌ i] m} G I G$ Ú.BABBAR U S.ARGAB $^{\text {mušen mu[n }}{ }^{\mathrm{n}}$ eme-sal-lì 1-niš SÚD ...]

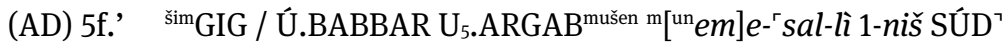

(xA) 2' [.............................. $]^{\ulcorner} \mathrm{U}_{5 .} \mathrm{ARGAB}^{\text {mušen }\urcorner}$

24 ND i 24 [ina MÚD $\left.{ }^{\text {gi] }}\right]^{5}$ EREN lu ina İ+GIŠ rgiš`EREN HE.HE Z[A.NA DÙ-uš ina A.MEŠ SÚD IGI.MIN-šú MAR]

(AD) 7’ ina MÚD EREN lu ina İ ER[EN H]E.HE ZA.NA DÙ-uš

(AD) 8' ina A.MEŠ SÚD MAR

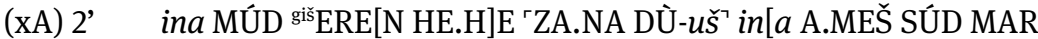

25 [šumma amēlu] ìnāšu šēra u dāma malâ murru [nīnû šammu peșû lipi kanakti]

26 rikibti arkabi sahlû šammū annûti [tēqìtu ša šèri āliki]

${ }^{25}$ If his eyes are full of tissue and blood: bitter-plant, [nīnû-mint, white plant, kanaktu-aromatic pith,]

${ }^{26}$ bat guano (and) sahlû-plant. These plants [are for an ointment (for the case when there is) a loose tissue (in the eyes).]

25 ND i 25 [DIŠ NA IG]I.MIN-šú UZU u MÚD DIRI.MEŠ šmS[ES?

(AD) 9f.' DIŠ NA IGI.MIN-šú UZU $u$ MÚD DIRI š [imS]ES úKUR.RA / Ú.BABBAR

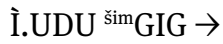

(bB) 3

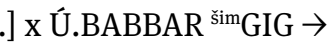

Overview of IGI 2 Manuscripts and Parallels: AA=BAM 20; AB=BAM 159; AD=BAM 22; AF=BAM 23; AG=BAM 15; bB=BAM 382; BoA=KUB 4/50; KA=CTN 4/123; NA=BAM 510; NB=BAM 513; NC=BAM 514; ND=BAM 515; NE=BAM 516; NI=BAM 480; NK=BAM 518; sA=IRAQ 65; $\mathbf{u A}=$ SpTU 50; $\mathbf{u B}=\mathrm{NBC}$ 4211; $\mathbf{x A}=\mathrm{CM} 37$ 
(uB) 16 [DIŠ LÚ] IGI.MIN.MEŠ-šú UZU u MÚD `DIRI.MEŠ ${ }^{2}$ ana ${ }^{\ulcorner T I}$ '-šú

$26 \mathrm{ND}$ i $26 \quad\left[\mathrm{U}_{5} . \mathrm{A}\right] \mathrm{RGAB}^{\text {mušen }}$ sah-lu-u Ú.MEŠ $a n-n u-t[i$............]

(AD) 10' U[5].ARGAB ${ }^{\text {mušen }}$

(AD) 11' sah-lé-e ina İ S[Ú]D MAR

(bB) $3 \quad \mathrm{U}_{5 .} \mathrm{ARGAB}^{\text {mušen }}$

(bB) $4 \quad[. .] \times.[$... İ] $]$ GIŠ HE.HE te-qit šá UZU DU

27 [šumma amēlu] ìnāšu qūqānī malâ gurušgaraš [ša šahî ...]

28 [ina] șubāti tessip sebet ūmē ina askuppi tetemmer [tušellâmma ...]

29 [...] malmališ $[$ tasâk ...]
${ }^{27}$ If a man's eyes are full qūqānu-'worms': ${ }^{28}$ you collect ${ }^{27}$ a 'vulva' of [a pig ... ${ }^{28}$ in a] cloth. You bury (it) under the threshold for seven days, [you remove (it) and ...] ${ }^{29}[$... you pound (it)] in equal measure $[\ldots]$

27 ND i 27 [DIŠ NA IGI.MI]N-šú qù-qa-ni DIRI.MEŠ gu-ru-uš-ga-r[a-aš ša šahî ...]

28 ND i 28 [ina TÚ]G te-sip 7 UD-me ina $\mathrm{KUN}_{4}$ te-te-m[er $\mathrm{E}_{11}-\mathrm{ma}$

29 ND i 29 [x x ma]l-má-l[iš SÚD x x x x ] x ${ }^{\ulcorner} \mathrm{x}^{\urcorner}[$

\section{GAP!}

Presumably more then 20 lines are missing!

50’ [... ina lipî] eșemti kurīti immeri ... [... tasâk teqqi]

51’ [...] malmališ tasâk ina dišpi himēti šaman erēni šuhti tasâk [teqqi]

\section{Alternative prescription 1}

52' ana ašri šanîmma qēm [ašnan] itti labti tasâk ana muhhi īnī̌u tašappak
${ }^{50}$ '... you pound (them) in marrow of $]$ a sheep's short [bone (and)] ... [... (and) daub (his) eyes.] ${ }^{51}$ You pound [...] in equal measures. You pound (them) in honey, ghee, cedar oil, copper patina. [You daub (his eyes with it)].

\section{Alternative prescription 1}

${ }^{52}$ Alternatively; you pound [cereal] flour with roasted grain, (and) you pour it onto his eyes.

Overview of IGI 2 Manuscripts and Parallels: AA=BAM 20; AB=BAM 159; AD=BAM 22; AF=BAM 23; AG=BAM 15; bB=BAM 382; BoA=KUB 4/50; KA=CTN 4/123; NA=BAM 510; NB=BAM 513; NC=BAM 514; ND=BAM 515; NE=BAM 516; NI=BAM 480; NK=BAM 518; sA=IRAQ 65; $\mathbf{u A}=$ SpTU 50; $\mathbf{u B}=\mathrm{NBC} 4211 ; \mathbf{x A}=\mathrm{CM} 37$ 
50' ND i 50' [............ ina İ.UDU GİR.PAD]. 'DU LÚGUD’.DA UD[U? ...] x x x [ SÚD MAR]

51’ ND i 51’ [x x] x [... má]l-ma-liš SÚD ina LÀL İ.NUN İ.GIŠ giš EREN SAHAR.URUDU S[ÚD? MAR]

52’ ND i 52’ 'DIŠ KIMIN' Zİ ' [ŠE.TIR] KI ŠE.SA.A SÚD ana UGU IGI.MIN-šú DUB

53’ šumma amēlu ìnāšu [... ina lipî kalīti] immeri iškūri ištēniš taballal ina lipî eșemti arikti tasâk teqqi
53'If a man's eyes are [...]: you mix together [... in kidney fat] of a [sheep] and wax. You pound (it) in marrow of a long bone (and) daub (his eyes).

53’ ND i 53’ DIŠ NA IGI.MIN-šú [... ina İ.UDU ÉLLAG UDU.NÍ]TA? GAB.LÀL 1-niš HE.HE ina İ.UDU GÌR.PAD.DU GÍD.DA SÚD te-eq-qí

(KA) 5’ [... ina A GAZI ${ }^{\mathrm{sa}}{ }^{\mathrm{r}} \mathrm{SILA}_{11}$ ina İ.UDU GÌR.PAD. 'DU ${ }^{\urcorner}$GÍD.DA SÚD M[AR]

54’ šumma ina īni [...] ìtenețtâ amēlu šù (šuātu) šipiršu ilabbirma īnāšu ippâ

55’ ana bulluțī̌su ištēn šiqil [...] ina himēti tasâk īnišu tēteneqqi
${ }^{54}$ 'If in the eye [...], (and both eyes) become progressively dark: the effect on this man will be long lasting and his eyes will be cloudy. ${ }^{55}$ In order to heal it: you pound one shekel of [...] in ghee (and) keep daubing his eyes.

54’ ND i 54’ DIŠ i-na IGI x [x x x] x i-te-né-ța-a NA BI KIN-šú SUMUN-ma IGI.MINšú ip-pa-a

55’ ND i 55’ ana TI-šú 1 GÍ[N x x x x ] x ina İ.NUN SÚD IGI.MIN-šú MAR.MEŠ

\begin{tabular}{|c|c|}
\hline $\begin{array}{l}\text { 56’ šumma amēlu ìnāšū nuppuhā [... qēm] } \\
\text { kukri qēm labti tahaššal tanappi ina } \\
\text { šikari tarabbak īnišu tașammid }\end{array}$ & $\begin{array}{l}\text { 56'If a man's eyes are inflamed [...]: you } \\
\text { crush and sift [... powder of] kukru- } \\
\text { aromatic (and) flour of roasted grain, } \\
\text { you make an infusion from (them) in } \\
\text { beer (and) bandage his eyes. }\end{array}$ \\
\hline
\end{tabular}

Overview of IGI 2 Manuscripts and Parallels: AA=BAM 20; AB=BAM 159; AD=BAM 22; AF=BAM 23; AG=BAM 15; bB=BAM 382; BoA=KUB 4/50; KA=CTN 4/123; NA=BAM 510; NB=BAM 513; NC=BAM 514; ND=BAM 515; NE=BAM 516; NI=BAM 480; NK=BAM 518; SA=IRAQ 65; $\mathbf{u A}=$ SpTU 50; $\mathbf{u B}=\mathrm{NBC}$ 4211; $\mathbf{x A}=\mathrm{CM} 37$ 


\section{Alternative prescription 1}

57’ '̌ammu peșû aban [gabî emesalli] šammū țīpu ša ìnī

\section{Alternative prescription 1}

57'White plant, gabû-[alum, emesallusaline solution]; drugs are (used as) eyedrops.

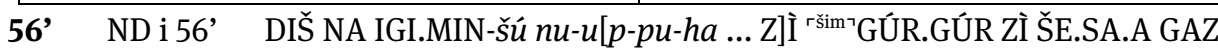
SIM ina KAŠ tara-bak IGI.MIN-šú LAL

57’ ND i 57’ Ú.BABBAR NA 4 [ga-bi-i ${ }^{\text {mun }}$ eme-sal-li] 'Ú.HI'.A ți-pu šá IGI.MIN

58’ šumma amēlu īnāšu barrā [dimta ukallā šamma peșâ] iškūra? [ina himēti tasâk]

59' sebet ūmē ina askuppi [tetemmer tušellâmma ...]
${ }^{58}$ 'If a man's eyes are flickering [and contain tear(s) you pound white plant (and)] wax? [in ghee ${ }^{59}$ You bury (the ingredients)] under the threshold for seven days, [you remove it and ...].

58' ND i 58’ DIŠ NA IGI.MIN-šú ba[r-ra u ÉR ú-kal-la ] ${ }^{\mathrm{GABB}}{ }^{?\urcorner} \cdot \mathrm{L}[\mathrm{A} L$ ? $]$ x $x$ $\mathrm{x}(\mathrm{x})$

(AB) iv 29f.' DIŠ NA IGI.MIN-šú bar-ra u ÉR ú-kal-la / Ú.BABBAR `LÀL ' ina İ.NUN SÚD IGI.MIN-šú MAR

(uA) r.2f. [DIŠ NA] IGI.MIN-šú i-bar-ru-ra u ÉR ú-kal-la Ú.BABBAR LÀL / [(x)] KUR.RA ina İ SÚD MAR

(xA) r.4 [DIŠ NA I]GI.'MIN?'-šú i-bar-ru-r[a u É]R 'ú-kal’-la Ú.BABBAR LÀL.KUR.RA ina İ.'GIŠ` S[ÚD MAR]

\section{9' ND i 59' 7 UD-me ina $\mathrm{KU}\left[\mathrm{N}_{4}\right.$ te-te-mer $\mathrm{E}_{11}-m a$...] $\mathrm{x}[\ldots]$}

\begin{tabular}{|c|c|}
\hline $\begin{array}{l}\text { 60’ šumma amēlu ìnāšru [...] tasâk ìnīšu } \\
\text { teqqi ... [...] }\end{array}$ & $\begin{array}{l}{ }^{60} \text { 'If a man's eyes are }[. . .] \text { you pound }[\ldots] \\
\text { and daub his eyes ... [...] }\end{array}$ \\
\hline
\end{tabular}

61’ šumma amēlu ìnāšu [arpā ...] ina šamni u šuhti [̌̌a tangussi tasâk ìnī̌̌u teqqi]

${ }^{61}$ 'If a man's eyes [grew dark: you pound ...] in oil and patina [of a copper tangussu-kettle and daub his eyes.]

Overview of IGI 2 Manuscripts and Parallels: AA=BAM 20; AB=BAM 159; AD=BAM 22; AF=BAM 23; AG=BAM 15; bB=BAM 382; BoA=KUB 4/50; KA=CTN 4/123; NA=BAM 510; NB=BAM 513; NC=BAM 514; ND=BAM 515; NE=BAM 516; NI=BAM 480; NK=BAM 518; $\mathbf{s A = I R A Q ~ 6 5 ; ~}$ $\mathbf{u A}=$ SpTU 50; $\mathbf{u B}=\mathrm{NBC}$ 4211; $\mathbf{x A}=\mathrm{CM} 37$ 


\section{\begin{tabular}{|c|c|}
\hline $\begin{array}{l}\text { 62' hașba labīra ša kibša [išû] ina šamni } \\
\text { šuhti tasâk teqqi dišip šadê [...] }\end{array}$ & $\begin{array}{l}{ }^{\mathbf{6 2}^{\prime}} \text { You pound an old fungus-[ridden] } \\
\text { potsherd in oil (and) copper patina (and } \\
\text { daub his eyes). You [mix] mountain } \\
\text { honey [in ... and you daub his eyes]. }\end{array}$ \\
\hline
\end{tabular} \\ 61’ ND i 61’ DIŠ NA IGI.MIN-šú $a[r-p a ~ . . . . . . .$.$] ina İ u$ SAHAR.UR[UDU ša ŠEN.TUR SÚD IGI.MIN-šú MAR]}

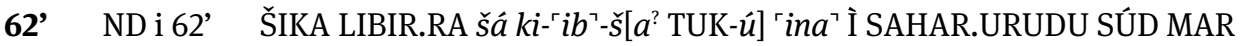
LÀL.K[UR.RA ...]

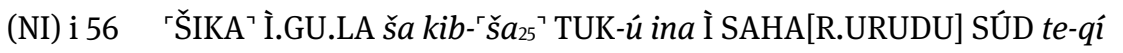

\section{3’ šumma amēlu ìnāšu șuhhutā karān šèlebi arqūssu tuhassa mêšu ina [diqāri tarabbak]}

64' pāna takattamma ša elēni išallu? ina šuhti u šamni [tasâk teqqi]

\section{Alternative prescription 1}

65’ ana ašri šanîmma sahlê ina šizbi takâṣ ina tinūri teppi ma'da lā ...[...]

66' lipi kanakti atā'iša aban gabî šammu peșâ kasâ ina himēti tasâk [īnišu teqqi]

\section{Alternative prescription 2}

67’ ana ašri šanîmma akal šibri turrar tasâk kīma țīpi tețeppi

\section{Alternative prescription 3}

68' ana ašri šanîmma qēm hallūri qēm kakkî qēm kasî sahlê qalâti balțāti ina diqāri tarabbak [tașammid]
63'If a man's eyes are under pressure; you squeeze fox-grape while still fresh, [you make an infusion from] its sap into a [diqāru-pot]. ${ }^{64}$ You cover the top, and whatever emerges? above, [you pound] in a copper patina and sesame oil [and daub (his eyes)].

\section{Alternative prescription 1}

${ }^{65}$ 'Alternatively: you grate a sahlû-plant into milk, you bake (it) in a tannouroven. It shall not dry out very much [...]. ${ }^{66}$ You pound kanaktu-aromatic pith, atā'išu-plant, alum, white plant (and) kasû-plant in ghee. [You daub his eyes with it].

\section{Alternative prescription 2}

67'Alternatively: you parch crumbled bread, you pound it (and) apply it as drops (on his eyes).

\section{Alternative prescription 3}

${ }^{68}$ 'Alternatively: you make an infusion from powder of chickpea, powder of lentil, powder of kasû-plant, freshly

Overview of IGI 2 Manuscripts and Parallels: AA=BAM 20; AB=BAM 159; AD=BAM 22; AF=BAM 23; AG=BAM 15; bB=BAM 382; BoA=KUB 4/50; KA=CTN 4/123; NA=BAM 510; NB=BAM 513; NC=BAM 514; ND=BAM 515; NE=BAM 516; NI=BAM 480; NK=BAM 518; sA=IRAQ 65; $\mathbf{u A}=\mathrm{SpTU} 50 ; \mathbf{u B}=\mathrm{NBC}$ 4211; $\mathbf{x A}=\mathrm{CM} 37$ 


\section{Alternative prescription 4}

69' ana ašri šanîmma ballukka kasâ ina šizbi tasâk īnišu teqqi

\section{Alternative prescription 5}

70’ ana ašri šanîmma šamma peșâ kutpâ turrar ina himēti tasâk teqqi :

\section{Alternative prescription 6}

70' ana ašri šanîmma lulâ ina lipî eșemti immeri tasâk teqqi

\section{Alternative prescription 7}

71' ana ašri šanîmma marat raqqi ina țābti tušnâl/tuštāl adi ablat tašakkan enūma ìtablu ina šamni šuhti tasâk teqqi

\section{Alternative prescription 8}

72' ana ašri šanîmma [marat? ...] eli patar siparri ina himēti tasâk teqqi

\section{Alternative prescription 9}

73’ ana ašri šanîmma šamma peșâ karān šēlebi [ina] šamni tanaddi ina ubānīka teqqi roasted sahlû-plant in a diqāru-pot. [You bandage (his eyes)].

\section{Alternative prescription 4}

${ }^{69}$ 'Alternatively: you pound ballukkuaromatic (and) kasû-plant in milk (and) you daub his eyes.

\section{Alternative prescription 5}

${ }^{70}$ Alternatively: you parch white plant (and) black frit, you pound (them) in ghee (and) daub.

\section{Alternative prescription 6}

${ }^{70}$ Alternatively: you pound lulu-antimony in the marrow of a sheep's bone (and) daub (his eyes).

\section{Alternative prescription 7}

${ }^{71}$ Alternatively: you cure the gall bladder of raqqu-turtle in salt, you leave (it) until it has dried out, when it has dried out you pound it in oil (and) copper patina (and) daub (his eyes).

\section{Alternative prescription 8}

${ }^{72}$ Alternatively: you pound [gall bladder of ...] over a bronze knife in ghee, (and) you daub (his eyes with it).

Alternative prescription 9

${ }^{73}$ Alternatively: you put white plant (and) fox-grape in sesame oil, you daub (his eyes) with your finger.

Overview of IGI 2 Manuscripts and Parallels: AA=BAM 20; AB=BAM 159; AD=BAM 22; AF=BAM 23; AG=BAM 15; bB=BAM 382; BoA=KUB 4/50; KA=CTN 4/123; NA=BAM 510; NB=BAM 513; NC=BAM 514; ND=BAM 515; NE=BAM 516; NI=BAM 480; NK=BAM 518; SA=IRAQ 65; $\mathbf{u A}=$ SpTU 50; $\mathbf{u B}=\mathrm{NBC}$ 4211; $\mathbf{x A}=\mathrm{CM} 37$ 
Alternative prescription 10

74' ana ašri šanîmma qaqqad șurāri tubbal tasâk [ina šamni tanaddi teqqi]

\section{Alternative prescription 11}

74' [ana ašri] šanîmma șurār igāri tubbal tasâk ina mušāli? ina pēmti tašakkan teqqi

\section{Alternative prescription 12}

75’ sikkat șēli immeri turrar tasâk (...)

UD.3.KAM ina mušāli tašakkan kayyamānu teqqi
Alternative prescription 10

${ }^{74}$ Alternatively: you dry a lizard's head (and) pound (it), [place it in oil (and) daub (his eyes)].

\section{Alternative prescription 11}

${ }^{74^{\prime}}$ Alternatively: you dry a wall-lizard (and) pound (it). You put (it) on a palette? over charcoal, (and) daub (his eyes).

\section{Alternative prescription 12}

${ }^{75}$ You parch a lamb chop (and) pound it. (...) Over three days you place (it) on a palette (and) you regularly daub (his eyes).

63’ ND i 63’ DIŠ NA IGI.MIN-šú șu-uh-hu-ta [GEŠTI]N.KA5.A SIG7-su tu-ha-sa A.MEŠ-šú ina d[uśUTUL tara-bak]

64' ND i 64' IGI DUL-ma šá AN.TA i-[ša] ?'-lu ina SAHAR.URUDU $u$ İ.GI[Š SÚD MAR]

65' ND i 65' DIŠ KIMIN ZÀ.HI.LI ina GA 'ta-ka'-aș ina NININDU te-ep-pi ma-'-da la $i-b a-x[\ldots]$

(AD) r.14f.'sah-lé-e ina G[A t]a-ka-a-aș / in[ $\left.a^{(\mathrm{im})}\right] \mathrm{S} U$.RIN.NA te-ep-pi ma- ${ }^{{ }^{r}}$ - $d a^{\urcorner} l a$ ir-ri-ra

(sA) ii 23ff. sah-lé-e / ki-șa-a-ti ina NININDU'(SUG) te-țe-eh-hi ma-'-du / la ir-ri-ra

66' ND i 66' İ.UDU ${ }^{\mathrm{sim}}$ GIG '́KUR.KU[R N]A 4 ga-bi-i Ú.BABBAR GAZI ${ }^{\text {sar }}$ ina İ.NUN SÚ[D IGI.MIN-šú MAR]

(AD) r.16' in [a] İ hal-și S[Ú]D MAR

(sA) ii 25f. ina İ.GIŠ.BUR tara-bak / IGI.MIN-šú MAR URUDU SUMUN ina İ.NUN.NA SÚD

(sA) ii $26 \quad$ GIN $_{7} r i b-k i$ IGI.MIN-šú MAR-ma TI- $u t t$

Overview of IGI 2 Manuscripts and Parallels: AA=BAM 20; AB=BAM 159; AD=BAM 22; AF=BAM 23; AG=BAM 15; bB=BAM 382; BoA=KUB 4/50; KA=CTN 4/123; NA=BAM 510; NB=BAM 513; NC=BAM 514; ND=BAM 515; NE=BAM 516; NI=BAM 480; NK=BAM 518; sA=IRAQ 65; $\mathbf{u A}=$ SpTU 50; $\mathbf{u B}=\mathrm{NBC}$ 4211; $\mathbf{x A}=\mathrm{CM} 37$ 
67’ ND i 67' [DIŠ K]IMIN NINDA ši-ib-ri t[ur-á]r SÚD ki-ma ți-pi te-țe-e[p-pi]

68' ND i 68' [DIŠ KIMI]N Zİ GÚ.GAL Zİ G[Ú.TUR] 'Zİ’ GAZI ${ }^{\text {sar }}$ sah-le-e qa-la-a-ti balța-ti ina dứUTUL tara-ba[k LAL]

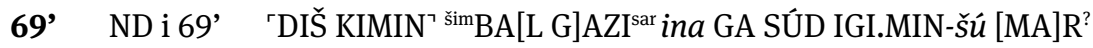

70' ND i 70' DIŠ KIMIN 'Ú'.BA[BBAR A]N.ZAH.GE 6 tur-ár ina İ.NUN SÚD MAR : DIŠ KIMIN KÙ.GAN ina İ.UDU GİR.PAD.DU UDU SÚD 'MAR'

71' ND i 71' DIŠ KIMIN ZÍ BAL.[G]I ${ }^{\ulcorner k u 67}$ ina MUN NÁ-al EN HÁD.DU GAR-an UD$m a$ HÁD.DU ina İ SAHAR.URUDU SÚD 'MAR'

(ND) iii 23’ [... EN HÁD.DU GAR-an U]D-ma HÂD.DU ina SAHAR.URUDU İ.NUN 'SÚD MAR'

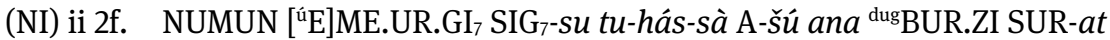
EN HÂD.DU GAR-an / U[D-m]a i-tab-lu ina İ SAHAR.URUDU SÚD MAR

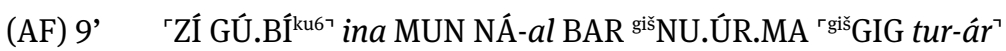

72’ ND i 72’ DIŠ KIMIN Z[í $\mathrm{x}(\mathrm{x} x)]$ UGU GÍR.ZABAR ina İ.NUN SÚD `MAR’ gloss:(?) x(?) $[\ldots]$

73’ ND ii 1 DIŠ KIMIN Ú.BABBAR "GEŠTIN.K[A $5_{5}$.A ina] İ?+GIŠ? ŠUB ina ŠU.SI-ka MAR

74’ ND ii 2 DIŠ KIMIN SAG.DU EME.ŠID HÁD.DU S[ÚD ina İ ŠUB MAR : DIŠ K]IMIN EME.ŠID É.GARs HÁD.DU SÚD ina ŠU.LÚ.ZABAR? ina NE GAR-an MAR

75’ ND ii 3 GAG.TI.TI UDU tur-âr SÚ[D (...) U]D.3.KÁM ina mu-šá-lì GAR SAG.UŠ MAR

Overview of IGI 2 Manuscripts and Parallels: AA=BAM 20; AB=BAM 159; AD=BAM 22; AF=BAM 23; AG=BAM 15; bB=BAM 382; BoA=KUB 4/50; KA=CTN 4/123; NA=BAM 510; NB=BAM 513; NC=BAM 514; ND=BAM 515; NE=BAM 516; NI=BAM 480; NK=BAM 518; sA=IRAQ 65; $\mathbf{u A}=$ SpTU 50; $\mathbf{u B}=\mathrm{NBC}$ 4211; $\mathbf{x A}=\mathrm{CM} 37$ 
76’ šumma amēlu īnāšu katmā x [... tasâk] ina himèti taballal ana libbi ìnī̌su tunattak

\section{Alternative prescription 1}

77’ muhha ša rīmti [tasâk? (...) ina] šamni u muhhi ša summati zikari

78' malmališ taballal UD.3.KAM [ina mušāli tašakkan] kayyamānu ana libbi innišu tattanaddi

\section{Alternative prescription 2}

79’ pel āribi [tasâk (...) arki] tušellâmma patar siparri himèta tapaššaš

80' [...] qātēka ina mê temessi

81' [... ina] himèti tulabbak teqqi
${ }^{76}$ 'If the man's eyes are closed: [you pound ...], mix (it) in ghee (and) you drip it into his eyes.

\section{Alternative prescription 1}

${ }^{77}$ You [pound?] wild cow's brain and [(...)] ${ }^{78}$ 'you mix (it) in equal measure ${ }^{77}$ in sesame oil and (in) the brain of a male pigeon. ${ }^{78}$ Over three days [you place (it) on a cosmetic palette (and)] you keep on applying it regularly into his eyes.

\section{Alternative prescription 2}

${ }^{79}$ '[You pound] a raven's egg, [(...) afterwards] you remove (it), and smear ghee on a bronze knife. ${ }^{80}[. .$.$] You wash$ your hands in water, ${ }^{\mathbf{8}}$ [....] You soften up ... [in] ghee and daub (his eyes with it).

The following prescriptions are fragmentary and mitght not be alternatives.

\section{Alternative prescription 3}

82’ [... ina mušāli tašakkan] kayyānam tèteneqqi

\section{Alternative prescription 4}

83' [...] tasâk teqqi

\section{Alternative prescription 5}

84' [...] ana šamni tarabbak rebiat kalgukki tasâk teqqi

\section{Alternative prescription 6}

85' [...] x SILAx $($ KISAL) kutpâ ina šaman erēni tasâk teqqi

\section{Alternative prescription 3}

${ }^{82}$ '[If, ... you place (it) on a cosmetic palette (and)] you keep on daubing (his eyes) regularly.

\section{Alternative prescription 4}

${ }^{83}[. .$.$] you pound, and daub (his eyes).$

\section{Alternative prescription 5}

${ }^{84}[. .$.$] you make an infusion from (it) in$ sesame oil, you pound one fourth measure of kalgukku-red paste (and) daub (his eyes).

\section{Alternative prescription 6}

${ }^{85}$ You pound [...] (and) x SILA-measures of black frit in cedar oil (and) daub (his eyes).

Overview of IGI 2 Manuscripts and Parallels: AA=BAM 20; AB=BAM 159; AD=BAM 22; AF=BAM 23; AG=BAM 15; bB=BAM 382; BoA=KUB 4/50; KA=CTN 4/123; NA=BAM 510; NB=BAM 513; NC=BAM 514; ND=BAM 515; NE=BAM 516; NI=BAM 480; NK=BAM 518; sA=IRAQ 65; $\mathbf{u A}=$ SpTU 50; $\mathbf{u B}=\mathrm{NBC} 4211 ; \mathbf{x A}=\mathrm{CM} 37$ 
Alternative prescription 7

86' $[. ..] \mathrm{x}$ :?

Alternative prescription 8

86’ karān šēlebi tasâk ina šamni ina tangussi tabbalakkat teqqi

\section{Alternative prescription 9}

87’ [...zēr] egemgïri urnê ištēniš tasâk teqqi

\section{Alternative prescription 10}

88' [...] ina šamni tasâk teqqi

\section{Alternative prescription 11}

89' [...] ... ana libbi ìnišu tunattak
Alternative prescription 7

${ }^{86}[\ldots] \mathrm{x}$ :?

\section{Alternative prescription 8}

${ }^{86}$ You pound fox-grape, (and) transfer (it) in oil into a copper tangussu-kettle, you daub (his eyes).

\section{Alternative prescription 9}

${ }^{87}[. .$.$] you pound together [... seeds of]$ egemgiru-plant, and urnû-plant (and) you daub (his eyes).

\section{Alternative prescription 10}

${ }^{88}[$...] you pound in oil, you daub (his eyes).

\section{Alternative prescription 11}

${ }^{89}[. .$.$] ... (and) you drip (it) into his eyes.$

76' ND ii 4 DIŠ NA IGI.MIN-šú DUL?-ma x [ $\mathrm{x}$ SÚ]D? ina İ.NUN HE.HE ana ŠÀ IGI.MIN-šú tu-na-tak

77’ ND ii $5 \quad$ UGU ša SÚN-tì S[ÚD? (...) ina] İ+GIŠ $u$ UGU ša TU TUư̌n $^{\text {NITA }}$

78 ND ii 6 mal-ma-liš HE.HE U[D.3.KÁM ina mu-šá-lì GAR S]AG.UŠ ana ŠÀ IGI.MIN-šú ŠUB.ŠUB-di

79’ ND ii $7 \quad$ NUNUZ UG[A ${ }^{\text {mušen }}$ SÚD x x x (x) EG]IR? E E1-ma GÍR.ZABAR İ.NUN ŠÉŠ

80’ ND ii $8 \quad x[$ [............................................................

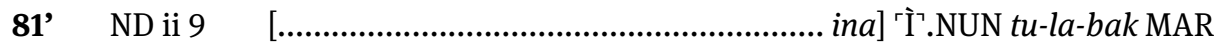

82' ND ii $10 \quad\left[\right.$................................... ina mu-šá-lì GAR-a]n ka-a-a-nam te-te-né-r qí ${ }^{7}$

83’ ND ii 11 ] 'SÚD? MAR

Overview of IGI 2 Manuscripts and Parallels: AA=BAM 20; AB=BAM 159; AD=BAM 22; AF=BAM 23; AG=BAM 15; bB=BAM 382; BoA=KUB 4/50; KA=CTN 4/123; NA=BAM 510; NB=BAM 513; NC=BAM 514; ND=BAM 515; NE=BAM 516; NI=BAM 480; NK=BAM 518; sA=IRAQ 65; $\mathbf{u A}=$ SpTU 50; $\mathbf{u B}=\mathrm{NBC}$ 4211; $\mathbf{x A}=\mathrm{CM} 37$ 
84' ND ii 12

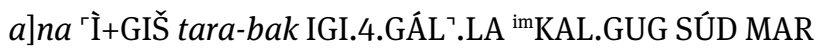

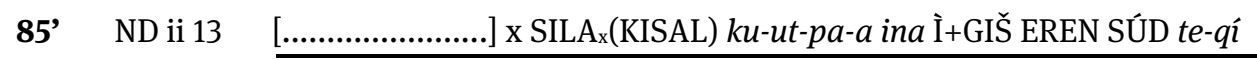

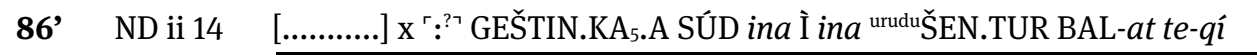

87’ ND ii 15

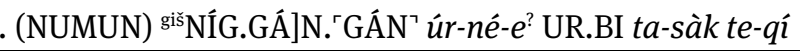

88, ND ii 16 ina İ SÚD te-qí

89' ND ii 17 .] x-ta ana ŠÀ IGI.MIN-šú tu-na-tak

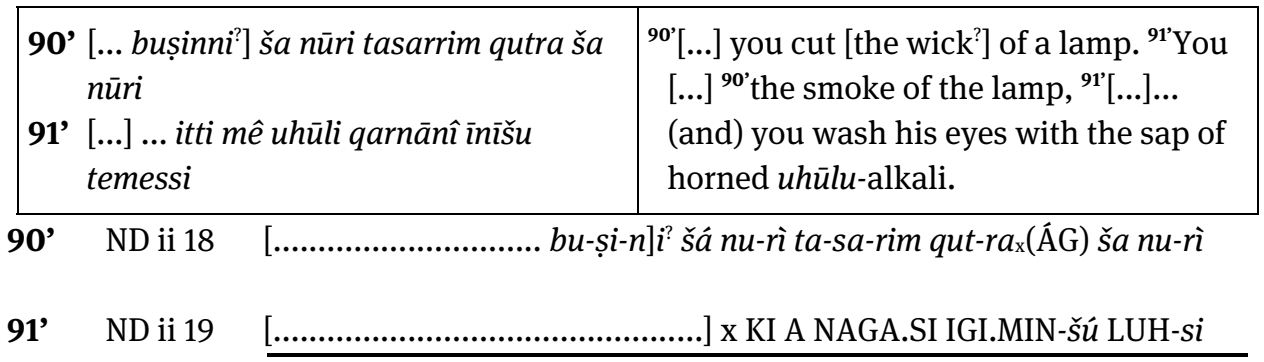

\begin{tabular}{|c|c|c|}
\hline 93 & $\begin{array}{l}\text { [... šamma peșâ] hamšat šiqil rikibti } \\
\text { arkabi malmališ taballal }\end{array}$ & $\begin{array}{l}{ }^{92}[\ldots] \text { you mix in equal measures [... white } \\
\text { plant], (and) five shekels of bat guano. } \\
{ }^{93}[. . .] \text { you pound (it with) a lead spoon in } \\
\text { oil and copper patina, daub (his eyes } \\
\text { with it). }\end{array}$ \\
\hline & \multicolumn{2}{|c|}{ 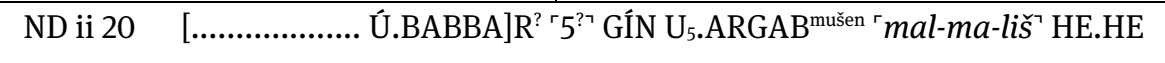 } \\
\hline & \multicolumn{2}{|c|}{ 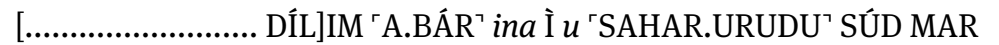 } \\
\hline
\end{tabular}

94’ [... ina] tangussi tukașșa ina tangussi taballal

${ }^{94}$ '....] you cool (it) off in a copper tangussu-kettle (and) you mix (it) in a copper tangussu-kettle. ${ }^{95}$ [...] you strip

Overview of IGI 2 Manuscripts and Parallels: AA=BAM 20; AB=BAM 159; AD=BAM 22; AF=BAM 23; AG=BAM 15; bB=BAM 382; BoA=KUB 4/50; KA=CTN 4/123; NA=BAM 510; NB=BAM 513; NC=BAM 514; ND=BAM 515; NE=BAM 516; NI=BAM 480; NK=BAM 518; sA=IRAQ 65; $\mathbf{u A}=$ SpTU 50; $\mathbf{u B}=\mathrm{NBC} 4211 ; \mathbf{x A}=\mathrm{CM} 37$ 


\begin{tabular}{|c|c|c|}
\hline $\begin{array}{l}105 \\
106 \\
107\end{array}$ & $\begin{array}{l}{[. . .] \text { īnīšunu ina șillî tutakkap }} \\
{[. . .] \text { šammī ša āribu ana șehrīšu ilqâa }} \\
{[. . .] \text { šâšu tasâk teqqi }}\end{array}$ & $\begin{array}{l}{ }^{105}[. . .] \text { you prick their eyes (of raven } \\
\text { chicks) with a needle. }{ }^{106}[. . . \text { (you take)] } \\
\text { the plants which the raven took to its } \\
\text { chicks (in order to heal them). }{ }^{107} \text { You } \\
\text { pound [... (these plants brought? }) \text { by it } \\
\text { (and) daub (his eyes). }\end{array}$ \\
\hline 5 & \multicolumn{2}{|c|}{ 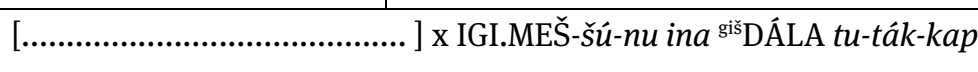 } \\
\hline 106' & \multicolumn{2}{|c|}{ 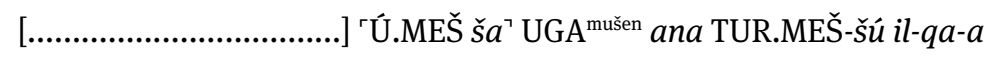 } \\
\hline 107' & \multicolumn{2}{|c|}{ 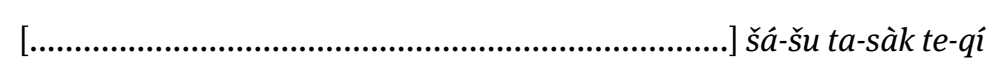 } \\
\hline
\end{tabular}

\begin{tabular}{|c|}
\hline $\begin{array}{l}\text { 108’ [...] kamkadi tasâk muhhi kurāri ša } \\
\text { kappi ìnīšu }\end{array}$ \\
\hline $\begin{array}{l}\text { 109' [tugallab (...)] ballukka zēr šammi } \\
\text { daqqiqi? zê upputte gūra ša qanî zēr } \\
\text { talupadi }\end{array}$ \\
\hline $\begin{array}{l}\text { 110’ larsīni ša halli lahri ša šumēli ša māšš } \\
\text { ūlidu }\end{array}$ \\
\hline $\begin{array}{l}\text { 111' [kalīt] lalî lakî ša šamma lā našku } \\
\text { utțata lā șabitu ina nemsēte taqallu }\end{array}$ \\
\hline $\begin{array}{l}\text { 112' balta ša țābti šarmada karānn šēlebi } \\
\text { zērr hațți rēề taqallu zēr lišāan [kalbi] }\end{array}$ \\
\hline $\begin{array}{l}\text { 113’ kurkānâ egemgīra šamma peșâ [qanâ] } \\
\text { țāba ištēniš tasâk taballal ina mê kasî } \\
\text { sekrūti }\end{array}$ \\
\hline $\begin{array}{l}\text { 114' kașûti talâš UD.15.KAM tașșanammid } \\
\text { UD.15.KAM šamma peșâ UD.3.KAM } \\
\text { teqqi }\end{array}$ \\
\hline
\end{tabular}

${ }^{108}$ '...] you pound kamkadu-plant. ${ }^{109} Y$ You [shave off] ${ }^{108}$ 'the surface of the kurārupustle of his eyelids. ${ }^{111}$ You roast in a tub ${ }^{109}$ [...], ballukku-aromatic, tiny? plant seeds, excrement of upputte-insect, reed leaf, seeds of talupadu-plant, ${ }^{110}$ 'a hoof of the left hind leg of an ewe which bore twins, ${ }^{111}$ '[kidney] of young suckling goat which has not (yet) bitten a plant nor grasped a grain kernel. ${ }^{112}$ You roast salted baltu-(thorn) plant, šarmaduplant, fox-vine (and) seeds of shepherd's-staff plant. ${ }^{113}$ You pound (and) mix together ${ }^{112}$ 'seeds of dog'stongue plant, ${ }^{113}$ 'kurkānû-plant, egemgiru-plant, white plant, sweet [reed]. ${ }^{114}$ You knead (all the abovementioned ingredients, first) ${ }^{113}$ 'in warmed-up (and then) ${ }^{114}$ 'cold ${ }^{113}$ 'sap of the kasû-plant. ${ }^{114}$ You continusly bandage (the eyes) for fifteen days. On the fifteenth day, you daub (his eyes) with white plant for three days.

108’ ND ii 36 k]am-ka-di SÚD UGU GIG.GIR ša kap-pi IGI.MIN-šú

Overview of IGI 2 Manuscripts and Parallels: AA=BAM 20; AB=BAM 159; AD=BAM 22; AF=BAM 23; AG=BAM 15; bB=BAM 382; BoA=KUB 4/50; KA=CTN 4/123; NA=BAM 510; NB=BAM 513; NC=BAM 514; ND=BAM 515; NE=BAM 516; NI=BAM 480; NK=BAM 518; sA=IRAQ 65; $\mathbf{u A}=\operatorname{SpTU} 50 ; \mathbf{u B}=\mathrm{NBC} 4211 ; \mathbf{x A}=\mathrm{CM} 37$ 
109' ND ii $37 \quad\left[t u^{?} \text {-gal?-lab? }\right]^{r u ́ B A L ~ N U M U N ~ U ' ~}\left[\right.$ da-a]q-qí-qí ŠE $\mathrm{E}_{10}$ up-pu-te gu-ra šá GI NUMUN úta-lu-pa-di

110’ ND ii $38 \quad[l a-a] r-s i ̀-n i ~ s ̌ a ~ h a l-l i{ }^{\ulcorner} \mathrm{U}_{8}{ }^{\urcorner}$ša GÙB ša MAŠ.TAB.BA Ù.TU

111 ND ii 39 [ÉLLAG] MÁŠ.TUR la-ki-i ša Ú la na- $a$ š-ku? ŠE.BAR la șa-bi-tú ina nem-se-te ta- ${ }^{\ulcorner}$qàl-lu?

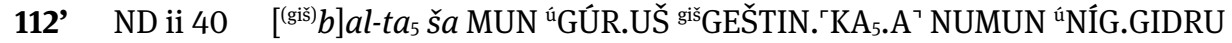
ta-qal-lu NUMUN úEM[E.UR.GI 7

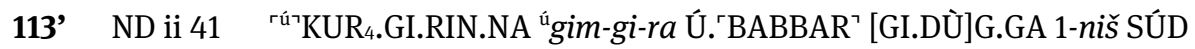
HE.HE ina A GAZI ${ }^{\text {sar }}$ sek-ru-t $[i]$

114' ND ii 42 [S]ED ta-là-aš UD.15.KÁM LAL.M[EŠ’] UD.15.KÁM Ú.BABBAR UD.3.KÁM te-eq-q[í]

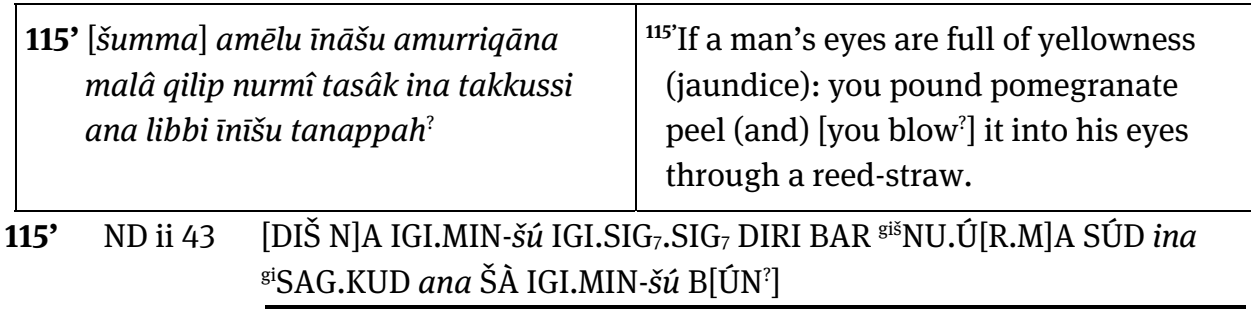

116' [šumma amēlu] īnāšu amurriqāna malâ hașab nurmî ina šamni tasâk teqqi : emesalli [tasâk teqqi']
${ }^{116}$ If a man's eyes are full of yellowness (jaundice): you pound pomegranate peel in oil and daub (his eyes) : [(or alternative) you pound] (it in) emesallusaline solution (and) [daub his eyes].

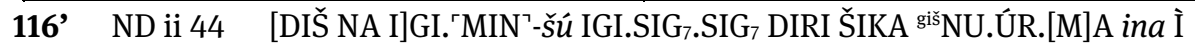
SÚD MAR : ${ }^{\text {mun }}$ eme-sal-lì S[ÚD? MAR?]

Overview of IGI 2 Manuscripts and Parallels: AA=BAM 20; AB=BAM 159; AD=BAM 22; AF=BAM 23; AG=BAM 15; bB=BAM 382; BoA=KUB 4/50; KA=CTN 4/123; NA=BAM 510; NB=BAM 513; NC=BAM 514; ND=BAM 515; NE=BAM 516; NI=BAM 480; NK=BAM 518; sA=IRAQ 65; $\mathbf{u A}=$ SpTU 50; $\mathbf{u B}=\mathrm{NBC} 4211 ; \mathbf{x A}=\mathrm{CM} 37$ 


\begin{tabular}{|c|c|}
\hline 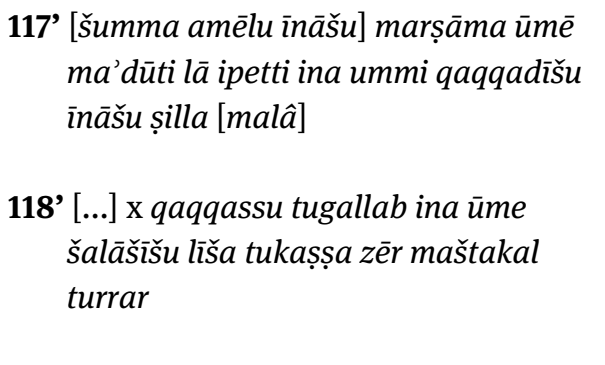 & $\begin{array}{l}{ }^{117} \text { 'If a man's eyes] are sick and he cannot } \\
\text { open (them) for many days, with his } \\
\text { head being feverish (ummu), (and) his } \\
\text { eyes are a film-[filled]. }{ }^{118} \text { [...] ... You } \\
\text { shave his head. You cool off (the head) } \\
\text { with dough three times a day. You parch } \\
\text { seeds of maštakal-plant, }{ }^{119} \text { you pound } \\
\text { [in ghee] on a lead [spoon] (and) daub }\end{array}$ \\
\hline $\begin{array}{l}\text { 119’ [ina himēti itqūr] abāri tasâk teqqi mê } \\
\text { temessi kasâ eli ìnīšu tašakkan adi } \\
\text { uzakkû tuttanār }\end{array}$ & $\begin{array}{l}\text { his eyes. You wash (the eyes) with water, } \\
\text { (and) you put kasû-plant over his eyes, } \\
\text { until they become clear (of film) you } \\
\text { keep on repeating (it). }\end{array}$ \\
\hline $\begin{array}{l}\text { Alternative prescription } 1 \\
\text { 120' }[\text { karān] šélebi dišip šadê ina himēti } \\
\quad \text { taballal tēteneqqi }\end{array}$ & 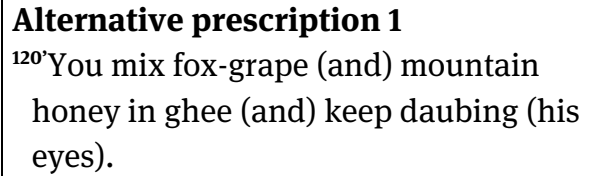 \\
\hline
\end{tabular}

117’ ND ii 45 [DIŠ NA IGI.MIN-šú G]IG-' ma UD $m a^{\urcorner-'-d u-t i ~ N U ~ ' B A D\urcorner ~ i n a ~ K U ́ M ~ S A G-~}$ šú IGI.MIN-šú GIS[SU DIRI?]

(NA) i 18’ [........ IGI.M]IN-šú GI[G .............. ma- ${ }^{-}$-d] $]$-ti NU BAD ina KÚM [....... ....]

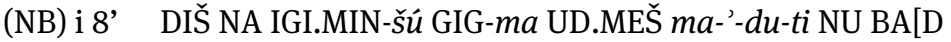

(NC) i 23'

$\check{S}] \hat{u}^{?}$

(AA) 11f.' ' 'DIŠ?? NA IGI.MIN-šú GIG-ma UD-me ME[Š] / NU BAD-te ina KÚM SAG.DU-šú 'IGI'.MI[N-šú] / 'GISSU DIRI ' $\rightarrow$

(AB) iv 3f.' DIŠ NA IGI.MIN-šú GIG-ma UD.MEŠ ma-'-du-te NU BAD-'te $e^{\urcorner}$/ ina KÚM SAG.DU-šú IGI.MIN-šú GISSU DIRI

118' ND ii 46 [... M]I? SAG.DU-su tu-gal-'lab ina UD-me 3-šú NÍG?.SILA 11. GÁ tu-kàṣ‘șa NUMUN úIN6.ÚŠ` $t[u r-a ̂ r]$

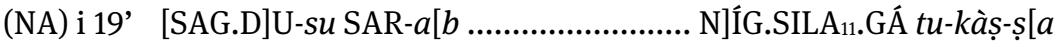

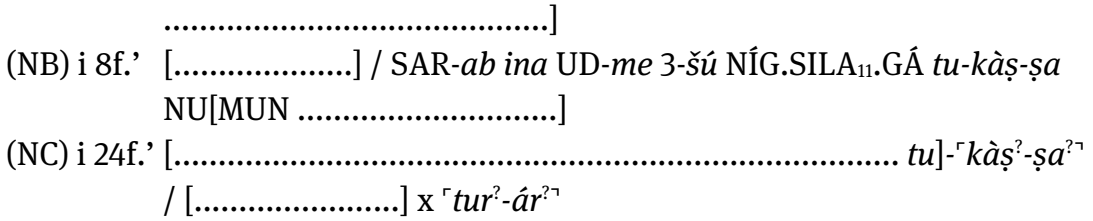

Overview of IGI 2 Manuscripts and Parallels: AA=BAM 20; AB=BAM 159; AD=BAM 22; AF=BAM 23; AG=BAM 15; bB=BAM 382; BoA=KUB 4/50; KA=CTN 4/123; NA=BAM 510; NB=BAM 513; NC=BAM 514; ND=BAM 515; NE=BAM 516; NI=BAM 480; NK=BAM 518; sA=IRAQ 65; $\mathbf{u A}=\operatorname{SpTU} 50 ; \mathbf{u B}=\mathrm{NBC} 4211 ; \mathbf{x A}=\mathrm{CM} 37$ 


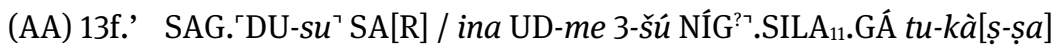
/ [NU]MUN úIN 6 .ÚŠ tur-ár $\rightarrow$

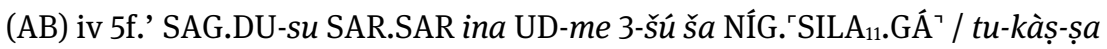
NUMUN úIN.NU.UŠ tur- $\left.{ }^{\ulcorner} a ́ r\right\urcorner$

119’ ND ii 47 [ina İ.NUN DÍLIM] 'A ' .BÁR SÚD MAR A LUH-si GAZI ${ }^{\text {sar }}$ UGU 'IGI.MINšú GAR? EN? ú-za-ku'-ú GUR.G[UR]

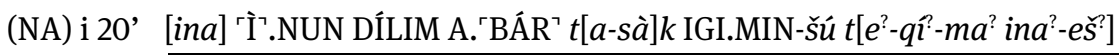

(NB) i 9f.' [...................] / DÍLIM A.BÁR ta-sàk IGI.MIN-šú [....

(NC) i 26' THE DIVIDING LINE IS NOT VISIBLE! in] $a^{?}-\left\ulcorner e s^{?} ?\right.$

(AA) 15ff.' ina İ.' NUN? / [DÍLIM] 'A ??.BÁR SÚD IGI.MIN-šú MAR.M[E(Š)] / [bul-ț] $u^{?}$ lat- $k\left[u^{?}\right]$

(AB) iv 7f.' ina İ.NUN DÍLIM A.BÁR SÚD IGI.MIN-šú / [M]AR.MEŠ-ma bul-țu lat-ku

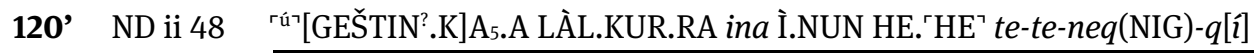

121' šumma amēlu ìnāšu marșāma šišìtu muhha lamassat ìnišu armat dimta illak īnāšu șilla malâma

122' errâ sahlāna zēr lišān [kalbi ...] šamma peșâ rikibti arkabi

123’ ištēššeret šammī šâšunu [ištēniš tasâk ina šaman] erēni taballal īnišu teqqīma iballuṭ
${ }^{121}$ 'If a man's eyes are sick, and a membrane covers the surface of the pupil of his eyes, tear(s) flow, (and) his eyes are film-filled: ${ }^{122}$ 'errû-colocynth, sahlânu-plant, seeds of [dog's] tongue plant [...], white plant, bat guano. ${ }^{123}$ 'Those eleven ${ }^{\text {sic! }}$ drugs you pound [together], mix [in] cedar [oil], daub his eyes, then he should recover.

121’ ND ii 49 DIŠ `NA IGI.MIN?-šú GIG-ma ši-ši-tu UGU dLAMA IGI.'MIN-šú á ár$m a] t$ [É]R.HI.A DU-ak IGI.MIN-šú GISSU DIRI-[ma]

(ND) ii 65 [DIŠ N]A I[GI.M]IN-[šú $\mathrm{x}-m a$ DUGUD ana TI-šú ILLU NU.LUH

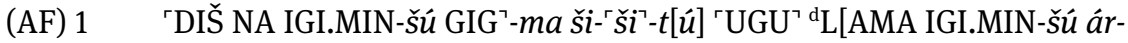
mat]

Overview of IGI 2 Manuscripts and Parallels: AA=BAM 20; AB=BAM 159; AD=BAM 22; AF=BAM 23; AG=BAM 15; bB=BAM 382; BoA=KUB 4/50; KA=CTN 4/123; NA=BAM 510; NB=BAM 513; NC=BAM 514; ND=BAM 515; NE=BAM 516; NI=BAM 480; NK=BAM 518; sA=IRAQ 65; $\mathbf{u A}=\mathrm{SpTU} 50 ; \mathbf{u B}=\mathrm{NBC}$ 4211; $\mathbf{x A}=\mathrm{CM} 37$ 
122’ ND ii 50 úrÚKUŠ`.LAGAB sah-la-na NUMUN úEM[E.UR.GI $\ldots$...] 'Ú'.BABBAR $\mathrm{U}_{5} \cdot \mathrm{ARGAB}^{\mathrm{m}[\mathrm{ušen}]}$

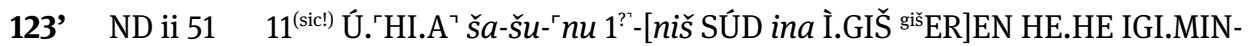
šú MAR-ma ${ }^{\ulcorner T I}{ }^{\urcorner}$

124’ šumma amēlu ìnāšu marșāma [šišītu muhha lamassat īnišu armat] digalšu kabit ana pūṣi ša muhhi lamassat īnišu nasāhi

125’ aktam [... tasâk ... ina šaman] erēni taballal ìnī̌u tèteneqqìma iballut
${ }^{124}$ 'If a man's eyes are sick, and [a membrane covers the surface of the pupil of his eyes], (and) his vision is 'heavy', in order to remove the white spot from the surface of the pupil of his eyes. ${ }^{125}$ 'You pound] aktam-plant, [... in] cedar [oil] you mix it, you keep daubing his eyes, and then he should recover.

124’ ND ii 52 DIŠ NA IGI.MIN-`šú GIG?-m[a ši-ši-tu UGU 'LAMA IGI.MIN-šú ár-mat

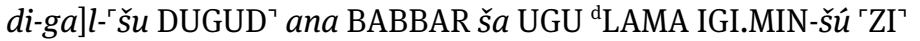

125’ ND ii 53 úat-ká[m ... SÚD ... ina İ] ${ }^{\text {rgišs }}$ EREN HE.HE IGI.MIN-šú MAR.MES-ma TI

\begin{tabular}{|c|c|}
\hline $\begin{array}{l}\text { 126’ šumma amēlu ìnāšu [marșāma ...] } \\
\text { digalšu kabit } \\
\text { 127’ ... [tasâk] innī̌u teqqi }\end{array}$ & $\begin{array}{l}\text { 126'If a man's eyes [are sick, and ....] his } \\
\text { vision is 'heavy', }{ }^{127} \text { ' [You pound ...] (and) } \\
\text { daub his eyes. }\end{array}$ \\
\hline
\end{tabular}

126’ ND ii 54 DIŠ NA IGI.MIN-'šú [GIG-ma ............................] x di-gal-šš 'DUGUD’

127’ ND ii 55 x x [..................................................... SÚ]D IGI.MIN-šú MAR

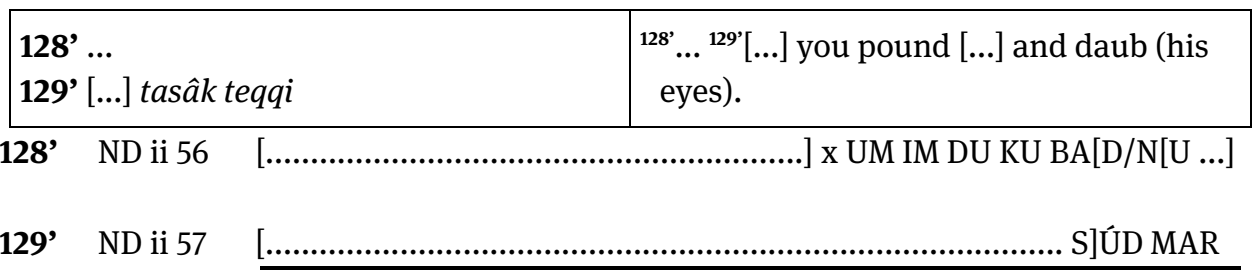

Overview of IGI 2 Manuscripts and Parallels: AA=BAM 20; AB=BAM 159; AD=BAM 22; AF=BAM 23; AG=BAM 15; bB=BAM 382; BoA=KUB 4/50; KA=CTN 4/123; NA=BAM 510; NB=BAM 513; NC=BAM 514; ND=BAM 515; NE=BAM 516; NI=BAM 480; NK=BAM 518; sA=IRAQ 65; $\mathbf{u A}=\operatorname{SpTU} 50 ; \mathbf{u B}=\mathrm{NBC} 4211 ; \mathbf{x A}=\mathrm{CM} 37$ 


\author{
130' [...] pillâ zikara \\ 131' [...] ikkalma ... [...] \\ 132' [... tasâk ina takkussi ana] libbi īnīšu \\ tanappah \\ 133’ [...] tasâk šanîš
}

\section{Alternative prescription 1}

134' [...] tanaddi ana muhhi hașab igulê

135' [...] tašarrap qutra ša šitti

136' [...] ahennâ īnišu tēteneqqīma iballuṭ
${ }^{130}\left[\right.$.... male pillû-plant. ${ }^{131}$ '.... he shall eat (it) and then ... [... $]^{132}[$... you pound it (and) through a reed-straw] you blow it [in] to his eyes. ${ }^{133^{\prime}}[. .$.$] you pound] and$ ditto (= you daub his eyes.)

\section{Alternative prescription 1}

${ }^{134}$ 'You] put [...]. You [(smear)...] on the surface of potsherd of fine salve, ${ }^{135^{\prime}}[\ldots]$ you burn it. The smoke of the remainder 136' [..]. You keep daubing his eyes separately, then he should recover.

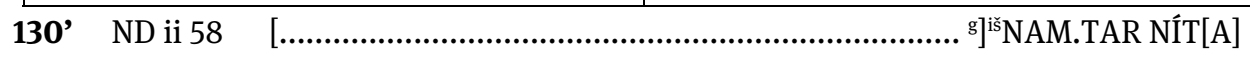

131’ ND ii 59 x $\mathrm{GU}_{7}-\max [(\mathrm{x})]$

132’ ND ii 60

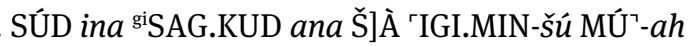

133’ ND ii 61 SÚ]D? MIN

134' ND ii 62 ŠUB-d]i ana UGU ŠIKA i-gu-le-e

135’ ND ii 63 .] x ta-šár-rap qut-ra šá šit-ti

136’ ND ii 64 a-he-e]n-na IGI.MIN-šú MAR.MEŠ-ma TI

137’ [šumma] amēlu [īnāšu marșāma] ...ma kabit ana bulluțišu hīl nuhurti

138' hìl baluhhi [... sebet] arhī u samānat arhī inassaha iballut
${ }^{137}$ [If] man's eyes [sick and ... ... and (the condition) is grave. In order to heal it: resin of nuhurtu-plant, ${ }^{138}$ 'resin of baluhhu-aromatic [... seven] or eight months will elapse, (then) he should recover.

\footnotetext{
137’ ND ii 65 [DIŠ N]A I[GI.M]IN-[̌̌́ ..........................................................] x-ma DUGUD ana TI-šú ILLU NU.LUH
}

Overview of IGI 2 Manuscripts and Parallels: AA=BAM 20; AB=BAM 159; AD=BAM 22; AF=BAM 23; AG=BAM 15; bB=BAM 382; BoA=KUB 4/50; KA=CTN 4/123; NA=BAM 510; NB=BAM 513; NC=BAM 514; ND=BAM 515; NE=BAM 516; NI=BAM 480; NK=BAM 518; sA=IRAQ 65; $\mathbf{u A}=$ SpTU 50; $\mathbf{u B}=$ NBC 4211; $\mathbf{x A}=\mathrm{CM} 37$ 
(ND) ii 49 DIŠ 'NA IGI.MIN'-šú GIG-ma ši-ši-tu UGU d'LAMA IGI.'MIN-šú á[r-ma]t [É]R.HI.A DU- $a k$ IGI.MIN-šú GISSU DIRI-[ma]

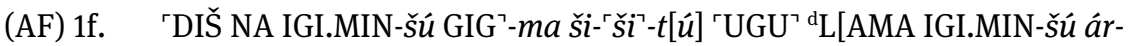
mat] / ' ana TI-šú ILLU NU.LUH ${ }^{\top}$

138’ ND ii 66 ILLU ${ }^{\text {rs̆im BULUH }}{ }^{\text {ú }}[$ 7 I]TI $u 8$ ITI ZI-ha TI

(AF) 2 ILLU šm

(AF) 3 'DIŠ NA 1 IGI.MIN-šú 'MÚ-ah GISSU ša 7 ITI [....................................]

139’ šumma amēlu ìnāšu šišitu [malâ ... hamiššer] uțțat hașbi šammi peșî ina himēti tasâk teqqi

\section{Alternative prescription 1}

140’ šumma amēlu šanîš ištēn šiqil rikibti [arkabi ina ... tasâk ...] tābīla tazarru

\section{Alternative prescription 2}

141’ šumma amēlu šanîš ištēn šiqil rikibti arkabi ina himēti tasâk teqqi : hamiššer uțtat hașbi [... ina ...] tasâk teqqi

\section{Alternative prescription 3}

142' imhur-lìm ina himēti [tasâk (ìnišu) teqqi]

\section{Alternative prescription 4}

143’ [... ištiššu] šinišu šalāšššu himēta tasâk teqqi
${ }^{139}$ 'If a man's eyes [are full of] a film, [...] you pound [15] grains of potsherd, (and) white plant in ghee, (and) daub (his eyes).

\section{Alternative prescription 1}

${ }^{140}$ 'If a man ditto: [you pound] one shekel of bat [guano in ...] (and) you spatter it in dry state (into his eyes).

\section{Alternative prescription 2}

${ }^{141}$ 'If a man ditto: you pound one shekel of bat guano in ghee and daub (his eyes) : you pound a 15 grain measure of potsherd [... (and) daub (his eyes).

\section{Alternative prescription 3}

${ }^{142}$ ' [You pound] imhur-lìm-plant in ghee [(and) daub (his eyes).]

\section{Alternative prescription 4}

${ }^{143}$ '... once], twice, three times, you pound ghee (and) daub (his eyes).

139’ ND ii 67 DIŠ NA IGI.MIN-šú ši-š[i-tú DIRI 1]5? ŠE ŠIKA Ú.BABBAR ina İ.NUN SÚD MAR

(AF) 4

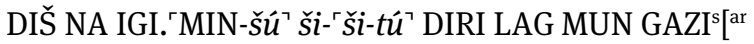

Overview of IGI 2 Manuscripts and Parallels: AA=BAM 20; AB=BAM 159; AD=BAM 22; AF=BAM 23; AG=BAM 15; bB=BAM 382; BoA=KUB 4/50; KA=CTN 4/123; NA=BAM 510; NB=BAM 513; NC=BAM 514; ND=BAM 515; NE=BAM 516; NI=BAM 480; NK=BAM 518; sA=IRAQ 65; $\mathbf{u A}=$ SpTU 50; $\mathbf{u B}=$ NBC 4211; $\mathbf{x A = C M} 37$ 
140' ND ii 68 DIŠ NA MIN 1 GÍN U[5.ARGAB ${ }^{\text {mušen }} \ldots . . . .$. SÚD ....... $]$ x [ $\left.\mathrm{x} t\right] a$-bi-la $a_{12}$ MAR

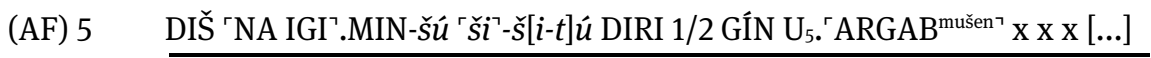

141’ ND ii 69 DIŠ NA MIN 1 GÍN U ${ }_{5}{ }^{\ulcorner}$.ARGAB ${ }^{\text {mušen }}$ ina İ.NUN SÚD MAR : 15 ŠE Š[IKA ... ina ... S]ÚD 'MAR`

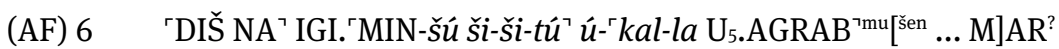

(AF) 7 '15 ŠE LÀL 7 KUR-e rSÚD` te-qé

142’ ND ii 70 úim-hur-lim ina İ.NUN [SÚD (IGI.MIN-šú) MAR]

(AF) 8 rú?im-hur-'lim? ina İ.NUN SÚD `te-qî

\section{GAP!}

143' ND iii 9' 1-šú] '2-šú 3-šú İ.NUN SÚD MAR'

NB.

The following prescriptions might be alternatives to the one on lines 139f.' This is, however, unclear since the diagnostic part is broken off.

144’ [... ina] himēti tasâk ina arhi šalāšǐšu teqqi rikibti arkabi ina himēti tasâk teqqi [arti] bìni arti èri

145’ [ina] țābāti talâš ìnī̌̌u tașammid šamma peșâ uhūla qarnānâ uzun lalî lā patān ana libbi īnī̌su tamazza'
${ }^{144}[. . .:]$ you pound in ghee (and) you daub (his eyes) three times in a month. You pound bat guano in ghee (and) daub (his eyes). ${ }^{145^{\prime}}$ You knead ${ }^{144^{\prime}}$ [leaf] of tamarisk, leaf of èru-tree ${ }^{145^{\prime}}$ [in] vinegar?, (and) you bandage his eyes. Without (him) eating, you squeeze out into his eyes white plant, horned uhūlu-alkali, (and) kid's-ear plant.

144’ ND iii 10’ [............ ina ] 'İ.NUN SÚD ina ITI 3-šú MAR U S.ARGAB $^{\text {mušen }}$ ina İ.NUN

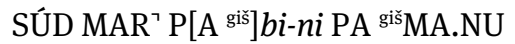

145’ ND iii 11' [ina A.GEŠTI]N.NA ta-la-aš IGI.MIN-šú LAL Ú.BABBAR NAGA.SI UR.TÁL.TÁL NU `pa-tan? [ana] `ŠÀ? IGI.MIN-šú ta-ma-za-a’

Overview of IGI 2 Manuscripts and Parallels: AA=BAM 20; AB=BAM 159; AD=BAM 22; AF=BAM 23; AG=BAM 15; $\mathbf{b B}=\mathrm{BAM}$ 382; BoA=KUB 4/50; KA=CTN 4/123; NA=BAM 510; NB=BAM 513; NC=BAM 514; ND=BAM 515; NE=BAM 516; NI=BAM 480; NK=BAM 518; sA=IRAQ 65; $\mathbf{u A}=$ SpTU 50; $\mathbf{u B}=\mathrm{NBC}$ 4211; $\mathbf{x A}=\mathrm{CM} 37$ 
(BoA) 3ff.' [BE KIMIN m]ar-șa-ma úÚR.TÁ[L.TÁL ...] / ta-na-za-a' ta-pa-[aš ...] / $a-n a$ ŠÀ IGI.MEŠ-šú $i-z[a$...]

\begin{tabular}{|c|c|}
\hline $\begin{array}{l}\text { 146' [...] ... emesalli sīkti labti taballal } \\
\text { turrar tasâk teqqi }\end{array}$ & $\begin{array}{l}{ }^{146^{\prime}}[. . .] \text {...: you mix emesallu-saline } \\
\text { solution (and) roasted grain powder, } \\
\text { you parch (the mixture), pound it (and) } \\
\text { daub his eyes. }\end{array}$ \\
\hline
\end{tabular}

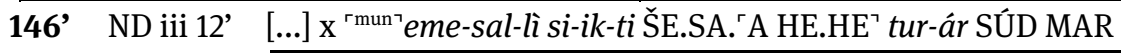

147’ [šumma ...] šuhta ina libbi lipi sāqi șabìti tasâk teqqi
${ }^{147}$ [If ...:] you pound copper patina into fat of a gazelle's thigh and daub (his eyes).

147’ ND iii 13' [DIŠ ........ SAH]AR.URUDU ina ŠÀ İ.UDU sa-a-qí M[AŠ.D]À SÚD MAR

(NI) i 44 sa]-a-qí MAŠ. 'DÃ SÚD? te-qí

\begin{tabular}{|c|c|}
\hline 148' [šumma ... murru ina šizbi musukkati & ${ }^{148^{\prime}}$ [If ...:] you pound [bitter-plant in milk \\
ša zikara] ūlidu tasâk teqqi dišpa & from a woman in maternity, who] gave \\
himèta ana libbi ìnǐšu tunattak & birth [to a male] (and) daub (his eyes), \\
& you drip into his eyes honey (and) ghee. \\
\hline
\end{tabular}

148’ ND iii 14’ [DIŠ ... šm SES GA munuś́.ZÚG šá NI]TA? Ù.TU SÚD 'MAR LÀL' İ.NUN.NA ana ŠÀ IGI.MIN-šú tu-na-ták

149’ [šumma ...] x x [...] šāru ul ușșâ 150' [...] ... ina himèti taballal teqqi
${ }^{149}{ }^{\prime}$ [If ....] x x [...] wind does not come out. ${ }^{150}[. .$.$] ... you mix [...] in ghee (and) daub$ (his eyes).

149’ ND iii 15’ [DIŠ .] x x [...] IM NU È- $a$

150' ND iii 16' ] $x$ ina İ.NUN HE.HE MAR

151' [...] tašaqqal teqqi ${ }^{151}$ '...] you weigh (it and) daub (his eyes). 151' ND iii 17' ta-ša-qàl MAR

Overview of IGI 2 Manuscripts and Parallels: AA=BAM 20; AB=BAM 159; AD=BAM 22; AF=BAM 23; AG=BAM 15; bB=BAM 382; BoA=KUB 4/50; KA=CTN 4/123; NA=BAM 510; NB=BAM 513; NC=BAM 514; ND=BAM 515; NE=BAM 516; NI=BAM 480; NK=BAM 518; sA=IRAQ 65; $\mathbf{u A}=\operatorname{SpTU} 50 ; \mathbf{u B}=\mathrm{NBC} 4211 ; \mathbf{x A}=\mathrm{CM} 37$ 
152' [... rikibti arkabi] tābīla ina himēti taballal ašar šanîmma

${ }^{152}$ [...], you mix [bat guano] in dry state in ghee (and) ditto.

152’ ND iii 18' $\left.\mathrm{U}_{5 .} \mathrm{ARGAB}^{\mathrm{m}}\right]^{\text {ušen }}{ }^{\mathrm{r}} t a-b i-l a_{12}$ ina ${ }^{\mathrm{I}} \mathrm{I} . \mathrm{NUN}$ HE.HE KIMIN

153’ [...] ubāni-ași arqūssu ina mê temessi tahaššal mêšunu

154' [...] tubbal šihiltu šuhta malmališ taballal [ina mê] temessi dišip šadê tasâk teqqi
${ }^{153}$ [...] you wash ubāni-ași-plant in water while still fresh, crush it (and) you ... (filter out) their saps, ${ }^{154}$ 'dry (it) out, ... (and) mix in equal measure the filtrate (with) the copper patina. You wash (it) [in water], pound mountain honey (and) you daub (his eyes).

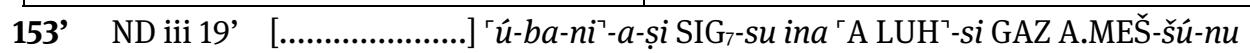

154’ ND iii 20’ [.....................] 'HÂD.DU 'ši-hi-il-tú SAHAR.URUDU mal-ma-liš HE.HE [ina A LU]H LÀL.KUR-e SÚD MAR

155’ [... ina] šizbi musukkati malmališ taballal tasâk teqqi
${ }^{155}$ '.... you mix in equal measure [... in the] milk from a woman in maternity, pound it (and) daub (his eyes with it).

155' ND iii 21' ina G]A munusÚ.ZÚG mal-ma-liš HE.HE 'SÚD` MAR

\section{6’ [...] mêšunu ana pursīti tașahhat \\ 157’ [... adi ablat tašakkan] enūma ìtablu ina šuhti himēta tasâk teqqi}

156’ ND iii 22’ $[\mathrm{SU}] \mathrm{R}-{ }^{\ulcorner} a t^{\top}$
${ }^{156}[. .$.$] you press out their sap into a$ pursitu-bowl. ${ }^{157}$ '... you leave it until it has dried], when it has dried out you pound it in copper patina (and) ghee, (and) daub (his eyes).

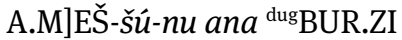

\section{7’ ND iii 23’ [... EN HÁD.DU GAR-an U]D-ma HÁD.DU ina SAHAR.URUDU İ.NUN} 'SÚD MAR $\urcorner$

Overview of IGI 2 Manuscripts and Parallels: AA=BAM 20; AB=BAM 159; AD=BAM 22; AF=BAM 23; AG=BAM 15; bB=BAM 382; BoA=KUB 4/50; KA=CTN 4/123; NA=BAM 510; NB=BAM 513; NC=BAM 514; ND=BAM 515; NE=BAM 516; NI=BAM 480; NK=BAM 518; SA=IRAQ 65; $\mathbf{u A}=$ SpTU 50; $\mathbf{u B}=$ NBC 4211; $\mathbf{x A}=\mathrm{CM} 37$ 
(ND) i 71' DIŠ KIMIN ZÍ BAL.[G]I I 'ku6? ina MUN NÁ-al EN HÁD.DU GAR-an UDma HÂD.DU ina İ SAHAR.URUDU SÚD 'MAR ${ }^{\top}$

(NI) ii 2f. NUMUN [ [́E]ME.UR.GI ${ }_{7} \mathrm{SIG}_{7}$-Su tu-hás-sà A-šú ana ${ }^{\mathrm{dug} B U R . Z I ~ S U R-a t ~}$ EN HÂD.DU GAR-an / U[D-m]a i-tab-lu ina İ SAHAR.URUDU SÚD MAR

158' [... ana] libbi īnī̌su tunattak lipâ ina šuhti tasâk teqqi
${ }^{158}$ '... into] his eyes you drip (it). You pound fat in copper patina (and) daub (his eyes).

158' ND iii 24' [........................ ana Š]À IGI.MIN-šú tu-na-ták İ.UDU ina SAHAR.URUDU SÚD 'MAR ${ }^{\urcorner}$

159’ [šumma ...] ìnāšu katmā kurșipti eqli ina mešēlti tasâk libbi īnī̌u

160’ [...] ... šadâna șābita šuruš gišimmari zikari

161' [...] gabâ ahê tasâk teqqi

159’ ND iii 25’ [DIŠ ŠÀ IGI.MIN-šú

160' ND iii 26’ ] ${ }^{\text {na4} K A . G I . N A . D A B . B A ~ S U H U S ̌ ~ g i s ̌ ~ G I S ̌ I M M A R ~ N I ́ T A ~}$

161’ ND iii 27’ IM.SAHAR.N]A4.KUR.RA $a$-he-e SÚD MAR

\begin{tabular}{|l|l|}
\hline $\begin{array}{l}\text { 162' [...] ... inib errî tahaššal ina šamni } \\
\text { tasâk teqqi }\end{array}$ & $\begin{array}{l}162^{\prime}[. . .: \text { you crush [...], (and) fruit of } \text { errû- } \\
\text { colocynth, pound it in oil (and) daub } \\
\text { (his eyes). }\end{array}$ \\
\hline
\end{tabular}

162’ ND iii 28' [.................................] x GURUN úÚKUŠ.LAGAB GAZ ina İ SÚD MAR

163’ [... ina dišip] šadê himēta šanî̌̌ ${ }^{163}[\ldots$ in] mountain [honey] (and) ghee, ditto.

Overview of IGI 2 Manuscripts and Parallels: AA=BAM 20; AB=BAM 159; AD=BAM 22; AF=BAM 23; AG=BAM 15; bB=BAM 382; BoA=KUB 4/50; KA=CTN 4/123; NA=BAM 510; NB=BAM 513; NC=BAM 514; ND=BAM 515; NE=BAM 516; NI=BAM 480; NK=BAM 518; sA=IRAQ 65; $\mathbf{u A}=$ SpTU 50; $\mathbf{u B}=\mathrm{NBC}$ 4211; $\mathbf{x A}=\mathrm{CM} 37$ 
163' ND iii 29' ina LÀ]L.' KUR'.RA İ.NUN MIN

164' [...] tasâk teqqi

${ }^{164}[\ldots]$ you pound (and) daub (his eyes).

164' ND iii 30' S]ÚD 'MAR ${ }^{\prime}$

\section{GAP!}

Break of approximately four lines!

\begin{tabular}{|c|c|}
\hline 165' [...] tasâk ina himēti [taballal ...] & ${ }^{165}$ You pound $[\ldots],[\mathrm{mix}]$ in ghee (and) $[\ldots]$ \\
\hline
\end{tabular}

165' ND iii 34' [..........................] 'SÚD ina İ`.N[UN HE.HE ....................]

\section{6’ [...] ìnī̌unu ina șillî tutakkap \\ 167' [...] ina itqūr abāri [tasâk teqqi]}

166’ ND iii 35’
${ }^{166}[. .$.$] you prick their eyes with a needle.$ ${ }^{167}[$... you pound ....] into a lead spoonsalve, [(and daub his eyes)] IGI.MEŠ]-šú-nu ina gišDÁLA $t[u-t a ́ k-k a p]$

167’ ND iii 36' .] ina DÍLIM A.BÁR [SÚD MAR]

\begin{tabular}{|c|l|}
\hline $\begin{array}{c}168 \\
\text { [...] billata tubbal tahaššal ina } \\
\text { himéti [tasâk teqqi] }\end{array}$ & $\begin{array}{l}\mathbf{1 6 8}^{\prime}[. . .] \text { you dry out beer mash (and) crush } \\
\text { (it) [...], (and) [pound] in ghee, [(and } \\
\text { daub his eyes)] }\end{array}$ \\
\hline
\end{tabular}

168' ND iii 37' . b]i-la-ta HÂD.DU GAZ ina İ.NU[N SÚD MAR]

\begin{tabular}{|c|c|}
\hline 169’ [...] tasâk ana libbi īnīšu tunattak & $\begin{array}{l}{ }^{169}[\ldots] \text { you pound }[. . .] \text {, you drip (it) into } \\
\text { his eyes. }\end{array}$ \\
\hline
\end{tabular}

169’ ND iii 38' [............................................... SÚ]D? ana ŠÀ IGI.MIN-šú t[u-na-ták]

\begin{tabular}{|l|l|}
\hline \multicolumn{1}{|c|}{ 170' [...] teqqi } & ${ }^{170}[\ldots]$, (and) you daub (his eyes). \\
\hline 170' ND iii 39'
\end{tabular}

\begin{tabular}{|c|c|c|}
\hline $171^{\prime}[\ldots] \ldots$ & $172,[\ldots] \ldots$ & ${ }^{171}[\ldots] \ldots{ }^{172}[\ldots] \ldots$ \\
\hline
\end{tabular}

Overview of IGI 2 Manuscripts and Parallels: AA=BAM 20; AB=BAM 159; AD=BAM 22; AF=BAM 23; AG=BAM 15; bB=BAM 382; BoA=KUB 4/50; KA=CTN 4/123; NA=BAM 510; NB=BAM 513; NC=BAM 514; ND=BAM 515; NE=BAM 516; NI=BAM 480; NK=BAM 518; sA=IRAQ 65; $\mathbf{u A}=$ SpTU 50; $\mathbf{u B}=\mathrm{NBC}$ 4211; $\mathbf{x A}=\mathrm{CM} 37$ 


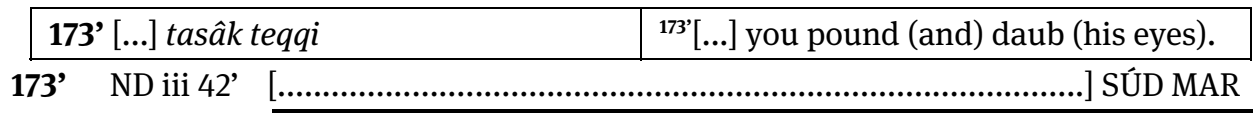

\begin{tabular}{|l|c|}
\hline $\begin{array}{l}\text { 174' [... ina] șubāti? tessip } \\
\text { 175' [... ana libbi īnīšu] tunattak }\end{array}$ & ${ }^{174}[\ldots]$ you collect [... in a] cloth. ${ }^{175^{\prime}}[. .]$. \\
\end{tabular}
174' ND iii 43' [........................................................................ ina TÚ $] \mathrm{G}^{\text {? }}$ te-sip

175’ ND iii 44' [................................................... ana ŠÀ IGI.MIN-šú tu]-na-ták

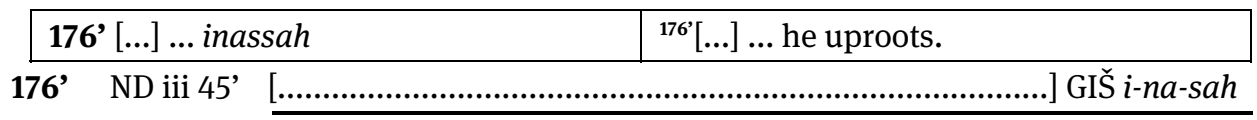

\begin{tabular}{|c|c|}
\hline $\begin{array}{l}\text { 177' [...] piqan șabìti } \\
\text { 178' [...] tasâk teqqi }\end{array}$ & $\begin{array}{c}{ }^{177}[\ldots], \text { (and) gazelle droppings, }{ }^{178}[\ldots] \\
\text { you pound [...], (and) daub (his eyes). }\end{array}$ \\
\hline \multicolumn{2}{|c|}{ 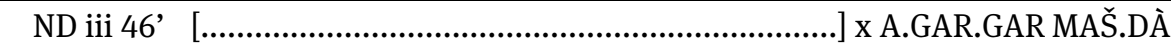 } \\
\hline ND iii 47' [...... & 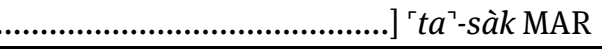 \\
\hline
\end{tabular}

\begin{tabular}{|c|c|}
\hline $179^{\prime}[\ldots] \ldots$. & ${ }^{179}[\ldots] \ldots$ \\
\hline
\end{tabular}

\begin{tabular}{|c|c|}
\hline 180’ [...] ina mê tasâk teqqi & $\begin{array}{l}\mathbf{1 8 0}^{\prime}[. . .] \text { you pound }[. . .] \text { in water, (and) } \\
\text { daub (his eyes). }\end{array}$ \\
\hline
\end{tabular}

180' ND iii 49' [.......................................................................] x ina A SÚD te-qi

\begin{tabular}{|l|l|}
\hline 181' [...] ina šikari talâš ìnišu tașammid & $\begin{array}{l}{ }^{181}[. . .] \text { you knead [...] in beer, (and) } \\
\text { bandage (his eyes). }\end{array}$ \\
\hline
\end{tabular}

181’ ND iii 50’ [............................................... in $] a^{\ulcorner}$KAŠ $\urcorner$ta-là-aš IGI.MIN-šú LAL

Overview of IGI 2 Manuscripts and Parallels: AA=BAM 20; AB=BAM 159; AD=BAM 22; AF=BAM 23; AG=BAM 15; bB=BAM 382; BoA=KUB 4/50; KA=CTN 4/123; NA=BAM 510; NB=BAM 513; NC=BAM 514; ND=BAM 515; NE=BAM 516; NI=BAM 480; NK=BAM 518; sA=IRAQ 65; $\mathbf{u A}=\operatorname{SpTU} 50 ; \mathbf{u B}=\mathrm{NBC} 4211 ; \mathbf{x A}=\mathrm{CM} 37$ 


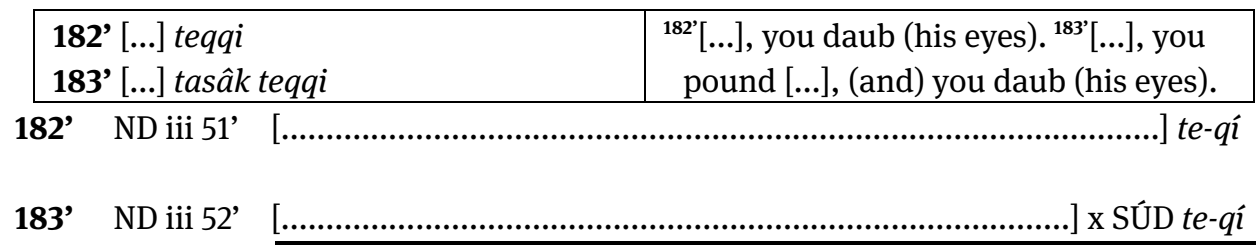

\begin{tabular}{|l|l|}
\hline $184^{\prime}[$ [... ina šamni tasâk teqqi & $\begin{array}{l}\text { 184}^{4}[. . .] \text { you pound [...] in oil, (and) you } \\
\text { daub (his eyes). }\end{array}$ \\
\hline
\end{tabular}

184’ ND iii 53’ [.......................................................................... in $]$ I İ SÚD te-qí

\begin{tabular}{|c|c|}
\hline 185' [... ina ïnišu $]$ marušti tummad & $185^{\prime}$. \\
\hline
\end{tabular}

\begin{tabular}{|c|c|}
\hline 186' [...] tasâk teqqi & $\begin{array}{l}{ }^{186^{6}}[. . .] \text { you pound [...], (and) daub (his } \\
\text { eyes). }\end{array}$ \\
\hline
\end{tabular}

186' ND iii 55' [.................................................................................... t t a-sàk te-qí

\begin{tabular}{|c|c|}
\hline $\begin{array}{l}\mathbf{1 8 7},[\ldots] \text { tasâk teqqi } \\
\mathbf{1 8 8} \mathbf{y}^{\prime . . .}[\ldots] \text { tarakkas }\end{array}$ & $\begin{array}{l}{ }^{187}[\ldots] \text { you pound }[\ldots], \text { (and) daub (his } \\
\text { eyes). }{ }^{188^{\prime}} . . .[\ldots] \text { you bind on. }\end{array}$ \\
\hline 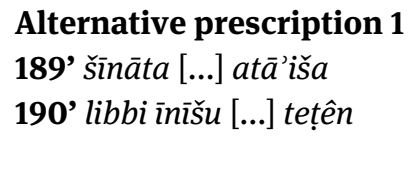 & $\begin{array}{l}\text { Alternative prescription } 1 \\
{ }^{189}\left[\text { You ...] urine, [... at ấ'išsu-plant, }{ }^{190^{\prime}} \text { the }\right. \\
\text { inner part of his eyes [...] you grind up } \\
{[\ldots . .]}\end{array}$ \\
\hline $\begin{array}{l}7^{\prime} \\
\text {, }\end{array}$ & SIUL \\
\hline
\end{tabular}

NB.

The following prescription 189f.' starts as an alternative one, but it is unclear if it refers to l. 187f.' or earlier.

189’ ND iii 58' 'KĀš’ [ .] ]'ं'KUR.KUR

190’ ND iii 59’ ŠÀ IGI.MI[N-šú . ÀR]A- ${ }^{r} n^{\urcorner}$

Overview of IGI 2 Manuscripts and Parallels: AA=BAM 20; AB=BAM 159; AD=BAM 22; AF=BAM 23; AG=BAM 15; bB=BAM 382; BoA=KUB 4/50; KA=CTN 4/123; NA=BAM 510; NB=BAM 513; NC=BAM 514; ND=BAM 515; NE=BAM 516; NI=BAM 480; NK=BAM 518; sA=IRAQ 65; $\mathbf{u A}=$ SpTU 50; $\mathbf{u B}=\mathrm{NBC}$ 4211; $\mathbf{x A}=\mathrm{CM} 37$ 


\begin{tabular}{|c|c|}
\hline $\begin{array}{l}\text { 191’ šumma ìnāšu [...] sahlê } \\
\text { 192’ šammi ašî [... ina] šizbi taballal } \\
\quad \text { teqqi }\end{array}$ & $\begin{array}{l}{ }^{191} \text { 'If his eyes are }\left[\ldots{ }^{192} \text { you .... }{ }^{191} \text { 'sahlû- }\right. \\
\text { plant, } \\
{ }^{192} \text { ' } a \text { šû-disease-plant, [...] you mix (the } \\
\text { ingredients) [in] milk, (and) daub (his } \\
\text { eyes). }\end{array}$ \\
\hline \multicolumn{2}{|c|}{ DIŠ IGI.MIN-šs $[\hat{~}$} \\
\hline \multicolumn{2}{|c|}{ 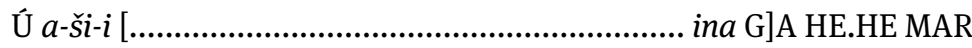 } \\
\hline
\end{tabular}

\begin{tabular}{|c|c|}
\hline $\begin{array}{l}\text { 193’ ana nuhhi [...] ina šamni taballal } \\
\text { teqqi }\end{array}$ & $\begin{array}{l}{ }^{193} \text { 'In order to soothe [...], you mix [...] in } \\
\text { oil (and) daub (his eyes). }\end{array}$ \\
\hline $\begin{array}{l}\text { Alternative prescription } 1 \\
\text { 194' lipâ hașab țābti ana libbi īnišu [...] } \\
\quad \text { ina šaman erēni taballal teqqi }\end{array}$ & $\begin{array}{l}\text { Alternative prescription } 1 \\
194^{\prime} \text { [You apply] fat and flake of salt into } \\
\text { [his] eyes, (and) you mix [...] in cedar } \\
\text { oil, (and) daub (his eyes). }\end{array}$ \\
\hline $\begin{array}{l}\text { Alternative prescription } 2 \\
\text { 195’ emesalli aktam tasâk ina dišpi [...] } \\
\quad \text { ina dišpi peșî patar siparri šanîš }\end{array}$ & $\begin{array}{l}\text { Alternative prescription } 2 \\
\text { 195’You pound emesallu-saline solution } \\
\text { (and) aktam-plant in honey, [you ...] in } \\
\text { honey. [...] in white honey with a } \\
\text { bronze knife, ditto (i.e. you mix and } \\
\text { daub his eyes). }\end{array}$ \\
\hline
\end{tabular}

193’ ND iii 62' a-na nu-úh-hi x [............................................... x ina İ HE.HE MAR

194’ ND iii 63’ İ.UDU ŠIKA MUN ana ŠÀ IGI.MI[N-šú ...] ina İ.GIŠ gišEREN HE.HE MAR

195’ ND iii 64’ ${ }^{\text {mun }} m e_{5}$-SILIM 'ak-tam SÚD ina LÀ[L ...] 'ina? LÀL BABBAR GÍR.ZABAR MIN

\begin{tabular}{|l|l|}
\hline $\begin{array}{l}\text { 196' šumma amēlu ìnāšu madirā [...] } \\
\text { šamma peșâ zēr GI.ZÚ.LUM.MA }\end{array}$ & $\begin{array}{l}\text { 196'If a man's eyes are rotten: [...] white } \\
\text { plant, seeds of 'date reed' }\end{array}$ \\
$\begin{array}{l}\text { Alternative prescription } 1 \\
\text { 197' rikibti arkabi [...] }\end{array}$ & $\begin{array}{l}\text { Alternative prescription } 1 \\
197\end{array}$ \\
\hline
\end{tabular}

Overview of IGI 2 Manuscripts and Parallels: AA=BAM 20; AB=BAM 159; AD=BAM 22; AF=BAM 23; AG=BAM 15; bB=BAM 382; BoA=KUB 4/50; KA=CTN 4/123; NA=BAM 510; NB=BAM 513; NC=BAM 514; ND=BAM 515; NE=BAM 516; NI=BAM 480; NK=BAM 518; sA=IRAQ 65; $\mathbf{u A}=$ SpTU 50; $\mathbf{u B}=\mathrm{NBC}$ 4211; $\mathbf{x A = C M} 37$ 


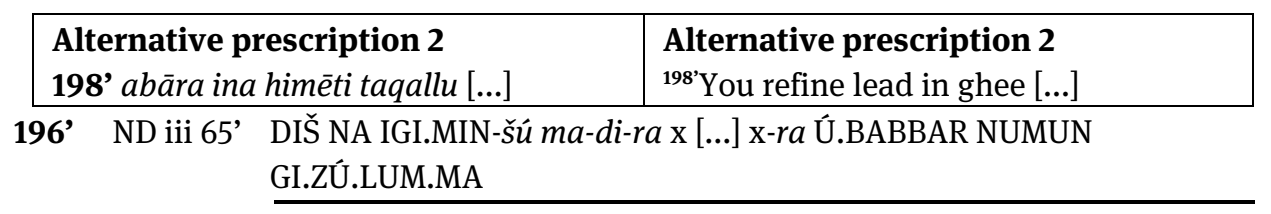

197’ ND iv $1 \quad \mathrm{U}_{5} \cdot \mathrm{ARGAB}^{\mathrm{mus}}[\mathrm{en}$

198’ ND iv 2 A.GAR ina İ.NUN BÍL x [....

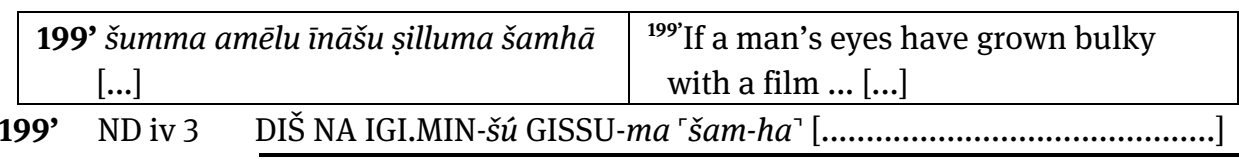

\begin{tabular}{|c|c|c|c|}
\hline \multicolumn{3}{|c|}{$\begin{array}{l}\text { 200' šumma amēlu ìn imittǐšu șillu[ma } \\
\text { šamhat ...] } \\
\text { 201' ina naglabi [šêli ...] }\end{array}$} & $\begin{array}{l}{ }^{200} \text { If a man's right eye has [grown } \\
\text { bulky] with a film [...] }{ }^{201} \text { 'with a [sharp] } \\
\text { scalpel [...] }\end{array}$ \\
\hline 0' & ND iv 4 & \multicolumn{2}{|c|}{ 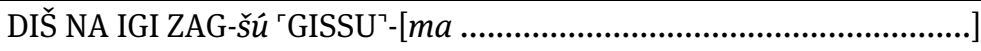 } \\
\hline 201' & ND iv 5 & \multicolumn{2}{|c|}{ 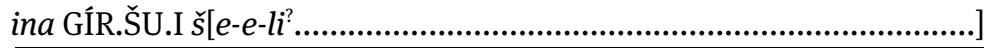 } \\
\hline
\end{tabular}

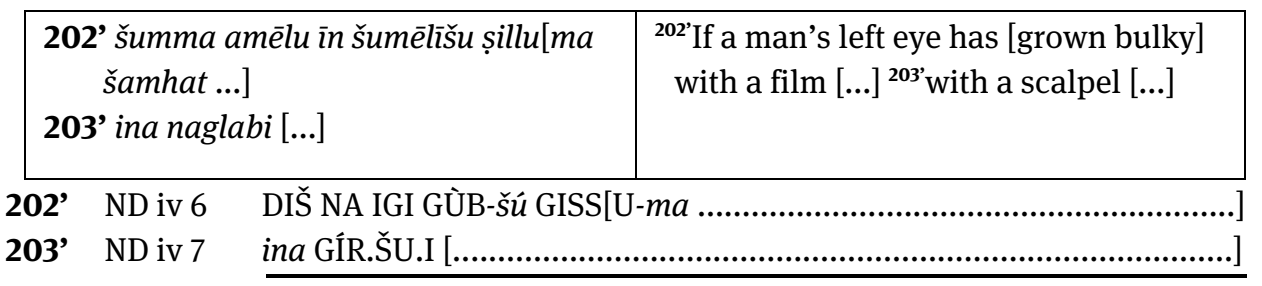

\begin{tabular}{|c|c|}
\hline $\begin{array}{l}\text { 204’ šumma amēlu ìnāšu marșā namrā } \\
\text { [...] }\end{array}$ & $\begin{array}{l}{ }^{204} \text { If a man's eyes are sick and shiny } \\
\text { [you ...] }\end{array}$ \\
\hline $\begin{array}{l}\text { Alternative prescription } 1 \\
\text { 205’ muhha ša erî ina šizbi musukkati } \\
\quad[\text { tasâk ...] }\end{array}$ & $\begin{array}{l}\text { Alternative prescription } 1 \\
{ }^{205} \text { 'YYou pound] the brains of an erû- } \\
\text { eagle in the milk from a woman in } \\
\text { maternity [...] }\end{array}$ \\
\hline
\end{tabular}

\footnotetext{
Overview of IGI 2 Manuscripts and Parallels: AA=BAM 20; AB=BAM 159; AD=BAM 22; AF=BAM 23; AG=BAM 15; bB=BAM 382; BoA=KUB 4/50; KA=CTN 4/123; NA=BAM 510; NB=BAM 513; NC=BAM 514; ND=BAM 515; NE=BAM 516; NI=BAM 480; NK=BAM 518; sA=IRAQ 65; $\mathbf{u A}=$ SpTU 50; $\mathbf{u B}=\mathrm{NBC} 4211 ; \mathbf{x A}=\mathrm{CM} 37$
} 


\begin{tabular}{|c|c|}
\hline $\begin{array}{l}\text { Alternative prescription } 2 \\
\text { 206' zēr GI.ZÚ.LUM.MA sīkti [labti } \\
\quad \text { taballal ...] }\end{array}$ & $\begin{array}{l}\text { Alternative prescription } 2 \\
{ }^{206}[\text { You mix] seeds of 'date reed' (and) } \\
\text { powder [of roasted grain ...] }\end{array}$ \\
\hline DIŠ NA IGI.MIN-šú & rZALAG.GA ${ }^{\top} \mathrm{x}[\ldots . . .$. \\
\hline
\end{tabular}

205’ ND iv 9 UGU ša $\mathrm{TI}_{8}{ }^{\text {mušen }}$ ina $\mathrm{GA}^{\text {rmunus }}{ }^{\text {?? }}$.[ZÚG? SÚD?

206’ ND iv 10 NUMUN GI.ZÚ.LUM.MA si-ik-t[i ŠE.SA.A HE.HE

\begin{tabular}{|c|c|}
\hline $\begin{array}{l}\text { 207’ šumma amēlu lamassāt īnišsu șilla } \\
\text { [malâ ...] } \\
\text { 208’ inī̌š teqqi ana ašri šanîmma hīl } \\
\text { abukkati [...] }\end{array}$ & $\begin{array}{l}{ }^{207} \text { 'If the man's pupils [are full] of a film, } \\
\text { [you .... }{ }^{\mathbf{2 0 8}} \text { you daub his eyes. Alter- } \\
\text { natively: resin of abukkatu-plant [...] }\end{array}$ \\
\hline
\end{tabular}

208’ ND iv 12 [I]GI.MIN-šú MAR DIŠ KIMIN ILLU L[I.TAR/DUR .

(AG) r.7 $\quad$ [DI]Š 'KIMIN $\urcorner$ ILLU LI.DUR [.....................................................]

\begin{tabular}{|c|c|}
\hline $\begin{array}{l}\text { 209' šumma ìn amēli șillu [șirihta mali } \\
\ldots . .] \\
\text { 210' [...] ... [...] }\end{array}$ & $\begin{array}{l}{ }^{209} \text { 'If a man's eye [is full of] a film (and) } \\
\text { [has inflammation .... }]^{210^{\prime}}[\ldots] \ldots[\ldots]\end{array}$ \\
\hline [DIŠ IG]I `LÚ’ GISSU `și & $-i h-t a_{5}$ DIRI .... \\
\hline
\end{tabular}

GAP!

Ca. 25 lines are missing!

\begin{tabular}{|c|c|c|}
\hline & $\begin{array}{l}{[. . .]} \\
{[. . .] \text {... ana ašri šanîmma }[. . .]}\end{array}$ & ${ }^{211}[\ldots] \ldots[\ldots]^{212}[\ldots]$.... Alternatively: $[\ldots]$ \\
\hline 11' & \multicolumn{2}{|c|}{ 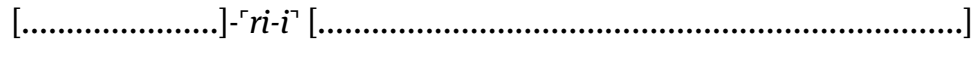 } \\
\hline 212' & \multicolumn{2}{|c|}{ 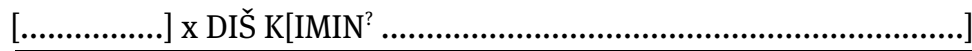 } \\
\hline
\end{tabular}

Overview of IGI 2 Manuscripts and Parallels: AA=BAM 20; AB=BAM 159; AD=BAM 22; AF=BAM 23; AG=BAM 15; bB=BAM 382; BoA=KUB 4/50; KA=CTN 4/123; NA=BAM 510; NB=BAM 513; NC=BAM 514; ND=BAM 515; NE=BAM 516; NI=BAM 480; NK=BAM 518; sA=IRAQ 65; $\mathbf{u A}=\mathrm{SpTU} 50 ; \mathbf{u B}=\mathrm{NBC} 4211 ; \mathbf{x A}=\mathrm{CM} 37$ 
213’ [šumma amēlu ìnāšu] ... malâ ... [...] 214' [...] dām kurșipti alpi șalmi u [...]

${ }^{213}$ '[If a man's eyes] are full of ... [...] ${ }^{214}$ [You .... ..., blood of a black ox-fly and $[. .$.

213' ND iv 43’ [DIŠ NA IGI.MIN-šú] $\left.\mathrm{x}{ }^{\ulcorner} \mathrm{x}\right\urcorner$ DIRI $\mathrm{x}$ [

214' ND iv 44' .] x MÚD kur-șib-ti $\mathrm{GU}_{4} \mathrm{GE}_{6} u$ x [...

\section{Catchline of Tablet Three}

215’ šumma amēlu ìnāšu dalhā dišpa peșâ ina himēti [ištēniš taballal ...]

\section{Catchline of Tablet Three}

${ }^{215}$ 'If a man's eyes are troubled: [you mix together] white honey in ghee (and) [...]

215’ ND iv 45’ [DIŠ NA] IGI.MIN-šú LÙ.LÙ LÀL BABBAR ina İ.[NUN 1-niš HE.HE ......]

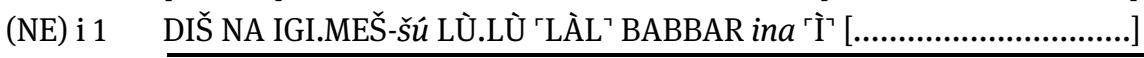

(NA) iv 31 [KA.INI]M.MA DIŠ N[A ................] L[Ù.LÙ]

(NC) iv 36 [.............] 'DIŠ NA IGI.MIN-šú LÙ.LÙ

(xA) r.9 [DI]Š NA IGI.MIN-šú LÙ.LÙ-`ha' LÀL İ.NUN.NA İ.'SAG' 1-niš HE.HE ina GE 6 IGI.MIN-šú DIR[I]

(uA) r.8f. DIŠ NA IGI.MEŠ-šú LÙ.LÙ-ha LÀL İ.NUN.NA İ.SAG gloss: hal-sa 1-niš x ERASURE? KÚM.KÚM-am ta-šá-hal ana IGI.MIN-šú ŠUB

\begin{tabular}{|c|c|}
\hline $\begin{array}{l}\text { Name of Tablet Two } \\
\text { 216’ šanû tuppu šumma amēlu [īnāšu } \\
\text { marșā] }\end{array}$ & $\begin{array}{l}\text { Name of Tablet Two } \\
\text { 216' Second [tablet] of the (treatise) 'If a } \\
\text { man's [eyes are sick'.] }\end{array}$ \\
\hline
\end{tabular}

\section{Ashurbanipal's Colophon, BAK no. 329}

217’ [ēkal Aššur-bāni-apli šar kiššati šar māt Aššur ša Nabû u Tašmētu uznu rapaštu išrukūšu

218'ēhuzzu ìnu namirtu nisiq tupšarrūti
217' Palace of Ashurbanipal king of the world, king of the Land Assyria, to whom Nabû and Tašmētu granted understanding, ${ }^{\mathbf{2 1 8}}$ [(who) acquired] clear insight [(and) a high level of scribal proficiency,] ${ }^{219}$ 'that skill which among the kings, my predecessor(s)

Overview of IGI 2 Manuscripts and Parallels: AA=BAM 20; AB=BAM 159; AD=BAM 22; AF=BAM 23; AG=BAM 15; bB=BAM 382; BoA=KUB 4/50; KA=CTN 4/123; NA=BAM 510; NB=BAM 513; NC=BAM 514; ND=BAM 515; NE=BAM 516; NI=BAM 480; NK=BAM 518; sA=IRAQ 65; $\mathbf{u A}=$ SpTU 50; $\mathbf{u B}=\mathrm{NBC}$ 4211; $\mathbf{x A}=\mathrm{CM} 37$ 


\begin{tabular}{|c|c|}
\hline $\begin{array}{l}\text { 219’ša ina šarrāni ālik mahrīya mamma } \\
\text { šipru [šuātu lā ēhuzzu] } \\
\text { 220' bulțī ištu muhhi adi șupri liqtī ahûti } \\
\text { tāhìzu nakla } \\
\text { 221'azugallūt Ninurta u Gula mala } \\
\text { bašmu ina tuppāni ašțrur asniq } \\
\text { abrēma } \\
\text { 222’ana tāmarti šitassīya qereb ēkallīya } \\
\text { ukīn }\end{array}$ & $\begin{array}{l}\text { no one [acquired]. }{ }^{221} \text { '[I wrote,] } \\
\text { checked, and collated tablets with } \\
{ }^{220} \text { 'medical prescriptions from cranium } \\
\text { to the (toe-) nail, non-canonical } \\
\text { material, elaborate teaching(s), (and) } \\
{ }^{221 '} \text { 'the advanced healing art(s) of } \\
\text { Ninurta and Gula, as much as created, } \\
\text { (and) }{ }^{222} \text { 'I placed (them) within of my } \\
\text { palace for consultation (and) my } \\
\text { reading. }\end{array}$ \\
\hline
\end{tabular}

217’ ND iv 47’ [É.GAL ${ }^{\mathrm{m}} A \check{s}$-šur-DÙ.A LUGAL ŠÚ 20 KUR AN.ŠÁR ${ }^{\mathrm{ki}}$ ša ${ }^{\mathrm{d}} \mathrm{AG} u^{\mathrm{d}}$ taš-me$t u_{4}$ GEŠTU.MIN ra-pa-âš-tu ${ }_{4}$ iš-ru-ku-šú]

218’ ND iv 48' [e-hu-uz-z]u [IGI.MIN n]a-'mir`-t[u ${ }^{\prime}$ ni-siq tup-šar-ru-ti]

219' ND iv 49' ša ina LUGAL.MEŠ-ni a-lik mah-`ri'-ia 'mám-ma šip-ru? [šu-a-tú NU ehu-uz-zu]

220’ ND iv 50’ ' 'bul'-ți TA muh-hi EN UMBIN 'liq-ti BAR.MEŠ ta’-h[i-zu nak-l]a

221' ND iv 51' a-zu-gal-lu-ut d Nin-urta u ${ }^{\mathrm{d}}$ Gu-la ma-la ba-ášs-mu 'ina ț tup-pa-a-ni ášsțùr as-n]iq IGI.KÁR-ma

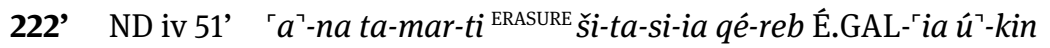

Overview of IGI 2 Manuscripts and Parallels: AA=BAM 20; AB=BAM 159; AD=BAM 22; AF=BAM 23; AG=BAM 15; bB=BAM 382; BoA=KUB 4/50; KA=CTN 4/123; NA=BAM 510; NB=BAM 513; NC=BAM 514; ND=BAM 515; NE=BAM 516; NI=BAM 480; NK=BAM 518; SA=IRAQ 65; $\mathbf{u A}=$ SpTU 50; $\mathbf{u B}=\mathrm{NBC} 4211 ; \mathbf{x A}=\mathrm{CM} 37$ 